\title{
DEVELOPMENT OF CELLPHONE INTEGRATED PARTICULATE MATTER POLLUTION MONITORING UNIT
}

\author{
by \\ Xuming Gao \\ Bachelor of Engineering, Ryerson University, 2015
}

\author{
A MRP \\ Presented to Ryerson University \\ in partial fulfillment of the \\ requirements for the degree of \\ Master of Engineering \\ in the Program of \\ Mechanical Engineering
}

Toronto, ON, Canada, 2015

(C) Xuming Gao 2015 


\section{Author's Declaration}

\section{AUTHOR'S DECLARATION FOR ELECTRONIC SUBMISSION OF A MRP}

I hereby declare that I am the sole author of this MRP. This is a true copy of the MRP, including any required final revisions.

I authorize Ryerson University to lend this MRP to other institutions or individuals for the purpose of scholarly research

I further authorize Ryerson University to reproduce this MRP by photocopying or by other means, in total or in part, at the request of other institutions or individuals for the purpose of scholarly research.

I understand that my MRP may be made electronically available to the public: 
Development of Cellphone Integrated Particulate Matter Pollution Monitoring Unit

Author: Xuming Gao

Master of Engineering in the Program of Mechanical Engineering, Ryerson University, 2015

\begin{abstract}
In this thesis, a miniature particulate matter detector is developed. The design utilizing light scattering (Mie Theory) phenomenon as the working principle measures mass concentration of particles with preselected size. The selection is realized by a special designed virtual impactor which collects particles within a range of desired physical sizes (10 micrometers or less). Theories corresponding to physical phenomenon are explained in detail. Parameter of each equation and procedure of design are elaborated.
\end{abstract}

The miniature PM monitoring unit achieves one-fifth of the size of the smallest commercial product in the market. All selected components (fan, laser, photodiode) are DC powered and lower wattage, which is made possible for phone battery as the power supply. Sensing range exceeds the worst PM10 reading in the history, and with sensitivity of $0.3 \mu \mathrm{g} / \mathrm{m}^{3}$, the air quality level can be divided into ten or more levels. Special designed virtual impactor separates PM10 from other air laden particles with low particle loss. 


\section{Acknowledgement}

Thank God for his grace and bless; this work would not have been possible without him.

To Prof. Siyuan He, my advisor, for his valuable comments, envisioned suggestions, and tireless guidance.

To my friends in the lab, Yuan Xue, Chao Fan, and Hui Zuo, for their recommends and ideas.

To my parents and my grandparents, for their unfailing love and unconditioned support.

To all of my friends in life who shared their priceless ideas. Special thank goes to H. Pishyar for his generous help. 


\section{Table of Contents}

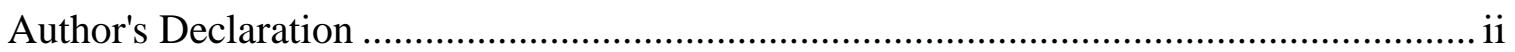

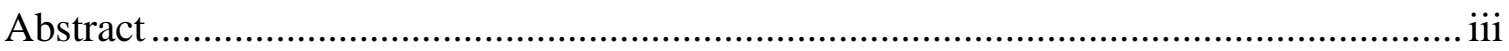

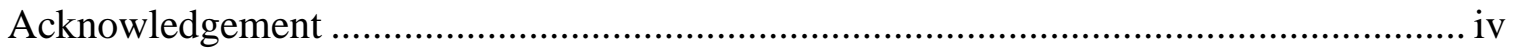

List of Figures ............................................................................................... vii

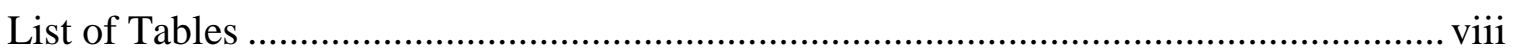

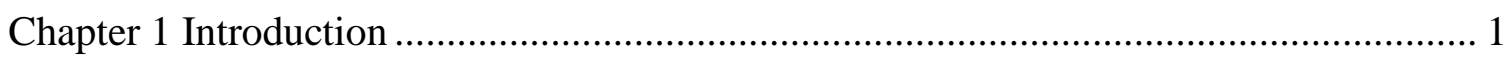

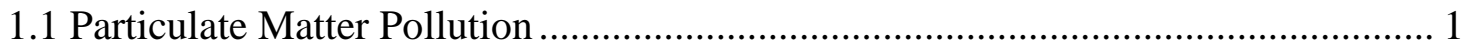

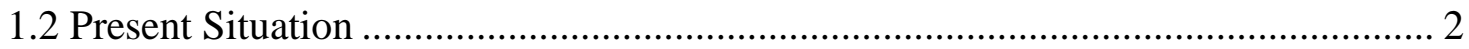

1.3 PM Monitoring Unit Integrated with a Cellphone................................................ 3

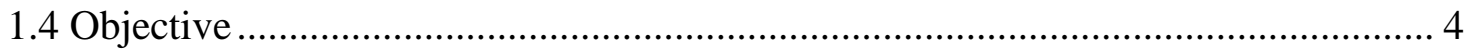

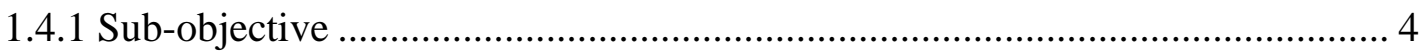

Chapter 2 Technology Review............................................................................ 5

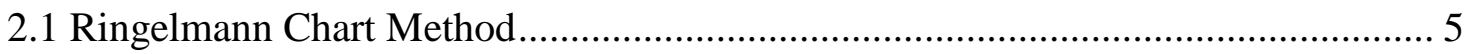

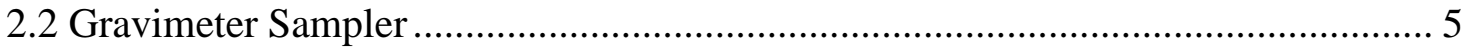

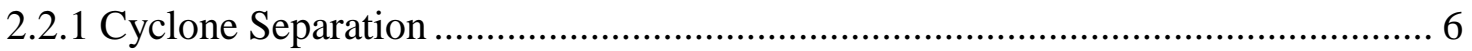

2.3 Thin-Film Bulk Acoustic Resonator (FBAR) ………………………………........ 7

2.4 Tapered Element Oscillating Microbalances ........................................................ 8

2.5 Light Absorption Method .................................................................................. 9

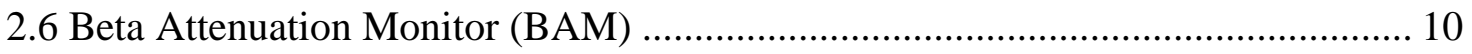

2.7 Charge Measurement ......................................................................................... 10

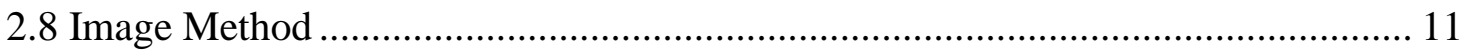

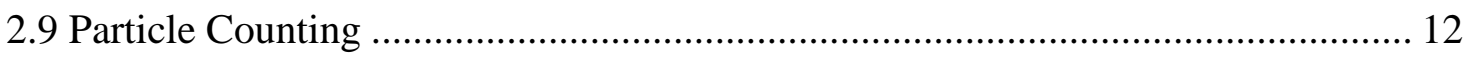

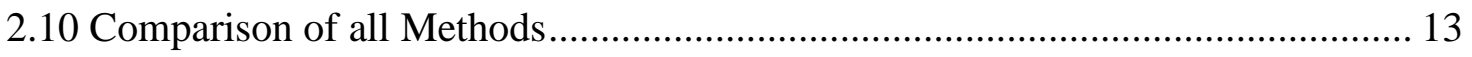

Chapter 3 Virtual Impactor Based Light Scattering Sensing Unit................................... 14

3.1 Working Principle ............................................................................................... 14

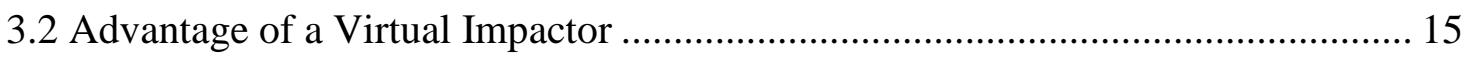

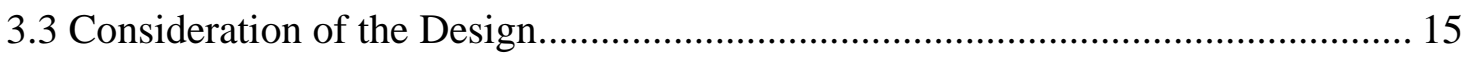

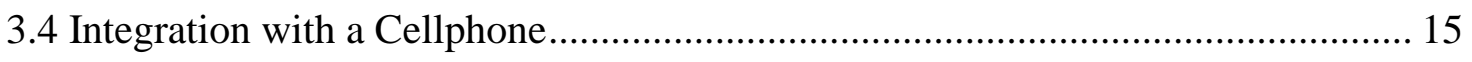

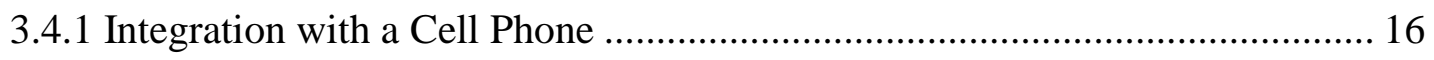

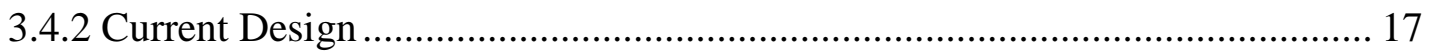




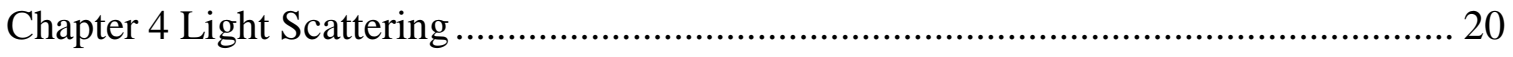

4.1 Light Scattering ............................................................................................. 20

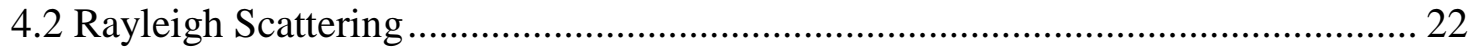

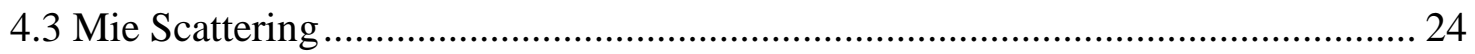

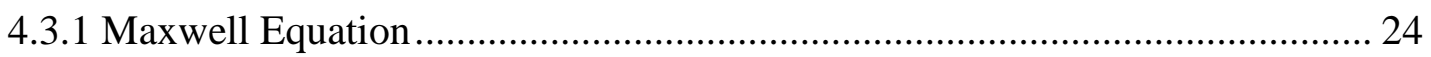

4.3.2 Mie Solution to Maxwell Equations .......................................................... 26

4.3.3 Resolution and Range of Detection Calculation by MatLab ........................... 29

Chapter 5 Virtual Impactor ...................................................................................... 31

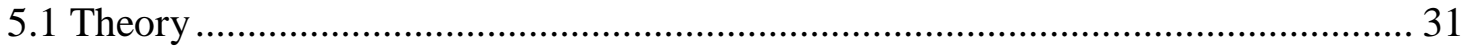

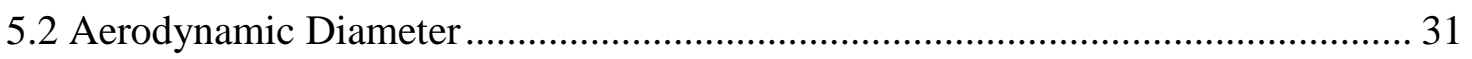

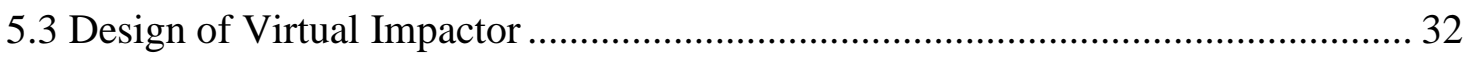

5.4 Particle Loss ..................................................................................................... 34

Chapter 6 Prototyping and Testing ……….............................................................. 36

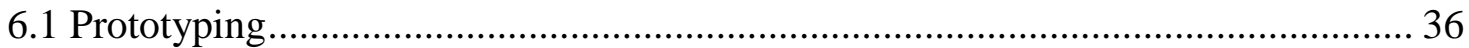

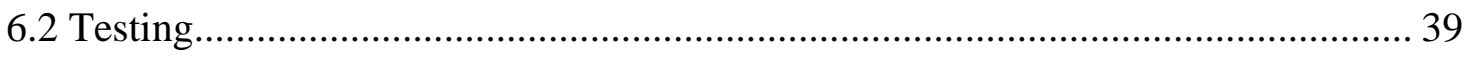

6.2.1 Uniform Sized Particle Experiments ................................................................ 40

6.2.2 Multiple Sized Particle Experiments .................................................................. 40

6.2.3 Idea of Testing Apparatus and Setup ............................................................. 40

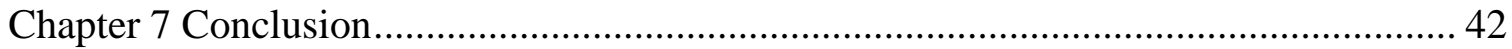

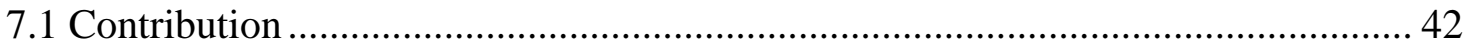

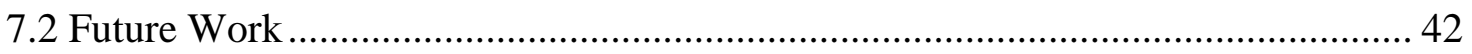

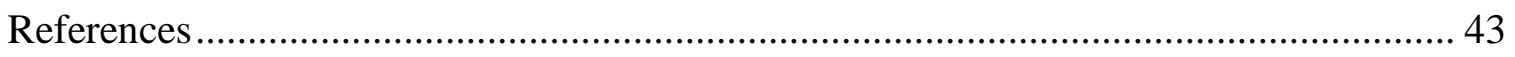

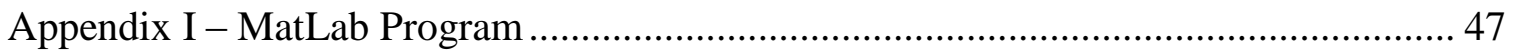

Appendix II - Selected Component Specification ........................................................... 52 


\section{List of Figures}

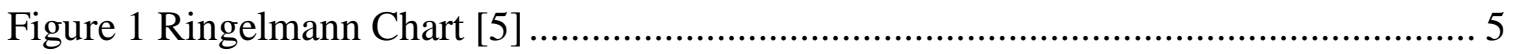

Figure 2 A Typical Cyclonic Separation Design [7] ........................................................ 6

Figure 3 Schematic of FBAR System (Incident UV/IR is used for discriminating the nature of particles) [9]............................................................................................ 8

Figure 4 Schematic of Image Method Setup [12] ....................................................... 11

Figure 5 A Taken Image of Particles [12] .................................................................. 12

Figure 6 Contour Plot of Particle Concentration by Image Process [12] ........................ 12

Figure 7 Work Flow of the PM10 Monitoring Unit ...................................................... 14

Figure 8 Schematic of Integration: as an Internal Component (Back Lid Removed) ...... 16

Figure 9 Schematic of Integration: as an Accessary ...................................................... 16

Figure 10 Photodiode Spectral Response Diagram [15] .............................................. 18

Figure 11 Light Scattering Due to Incident EM Wave [18] ........................................... 20

Figure 12 Visualization of Light Scattering by a Small Particle [20]............................. 21

Figure 13 Scattered Light Patten by Different Size of Particles [21] .............................. 22

Figure 14 Virtual Impactor Cross-section View [33] ..................................................... 33

Figure 15 (a) Grid used to determine particle trajectory in virtual impactor and (b)

Particle trajectory when entering by different streamline at the base condition [30] ....... 35

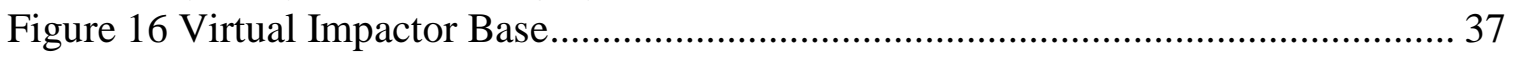

Figure 17 Virtual Impactor Cover (Flipped)............................................................... 37

Figure 18 Virtual Impactor Cover (Section View) ………............................................. 38

Figure 19 Assembly of Virtual Impactor ..................................................................... 38

Figure 20 Explode View of Assembly of Virtual Impactor (Base and Cover) along with Laser Diode (Collimated) and Photodiode ……………............................................. 39

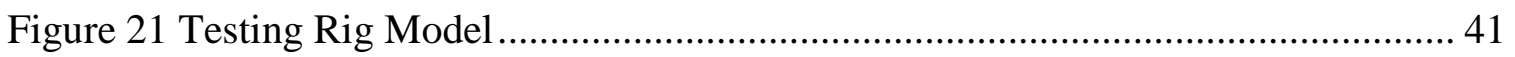

Figure 22 MatLab Program for Sensitivity Calculation .................................................. 47

Figure 23 Command for Calculating the Median of Sensitivity and its Result ................ 47

Figure 24 MatLab Program for Calculation of Upper Boundary of Sensing Range ........ 48

Figure 25 Command for Calculating the Median of Upper Boundary of Sensing Range

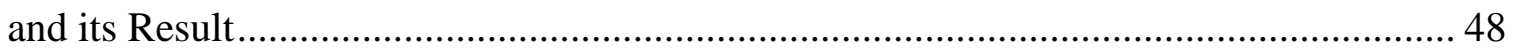

Figure 26 MatLab Program for Mie Coefficient Calculation [26] ................................... 49

Figure 27 MatLab Program for Mie Angular Functions [26] ......................................... 50

Figure 28 MatLab Program for Scatter Amplitude Calculation [26]............................... 51

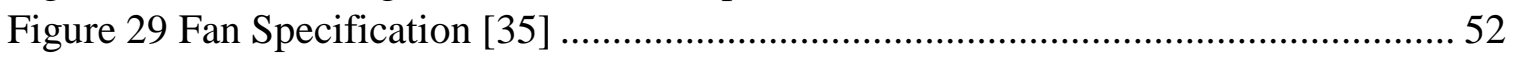

Figure 30 Photodiode Specification [15] ................................................................... 53

Figure 31 Photodiode Chart and Dimension Schematic [15] ......................................... 54

Figure 32 Schematic of a Low Noise Light-Sensitive Preamplifier Circuit by Hamamatsu

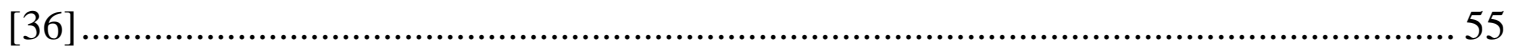

Figure 33 Laser Module Specification [37] .......................................................... 56 


\section{List of Tables}

Table 1 Air Quality Standard Breakpoint by EPA [12] ............................................... 3

Table 2 Dimension and Power of Each Component ......................................................... 36 


\section{Chapter 1 Introduction}

Human beings, just like all other creatures: vulnerability is one character shared among all. There are too many ways to end a life: suicide, murder, death by car crush, death by falling and many more. All these seem to happen within a blink of an eye, but there are other causes which can take weeks, months or even years to kill a person. Cancer may be one of the most well-known diseases that makes a person suffer for years until the last breath of one's life.

Creativity is one character that draws a line between humans and other animals: humans are capable of inventing things that never exist in the world for their own wellbeing. Even with this talent, human beings still have to vigorously protect themselves from different causes, which may surprisingly be the effects of our actions. There are too many designs for safety to list them all here. Among them, some designs make life less enjoyable, which sounds contradictory to their purpose of design, but they keep us safe. Seatbelt, helmet, and bullet proof vest are all amazing inventions and prefect examples that are considered less enjoyable. There are dangers out there and it is not as challenging to be protected from what can be seen, heard, touched, smelled, tasted, or even felt. Sadly, there are also hazards that cannot be sensed or are hardly sensed by human beings without any aid, for example: airborne particulate matters. Air does not only contains $74 \%$ nitrogen plus $25 \%$ oxygen, but also other solid particles in the order of microns to submicrons and consist of toxins that are too small to be detected by humans.

\subsection{Particulate Matter Pollution}

Particulate matter (PM) has become a daily focus in some the countries because its affect is too server to ignore. PM10 particularly, standing for all particulate matter whose size is less or equal to 10 micrometers, raises extensive attention. Chain structures enable adsorption capacity considerably to particulate matter. Two grams black carbon can cover a basketball court if it is fully expanded. $90 \%$ of the airborne particulate matters whose sizes greater than $10 \mu \mathrm{m}$ (PM10 above) are intercepted by nose hair and mucus. PM10 could travel along the respiratory system into the lung and reduce the 
functionality of immune system. The aerosols with their sizes are equal or smaller than $0.5 \mu \mathrm{m}$ (PM0.5) can travel through cell walls and gives rise to blood clots and myocardial infarction (MI). [1]

Particulate matter is a complex combination of small solid particles and liquid droplets that include dust, acid, organic chemicals and metals. Many participated material are toxins. Most toxins can have chemical reactions with other toxins, and makes it even more toxic [1]. Particles emit directly from a source, such as construction sites, fields, and fires, are known as primary particles. Others produced through chemical reactions are called secondary particles such as hydrocarbons $(\mathrm{HC})$, sulfur dioxides $\left(\mathrm{SO}_{2}\right)$ and nitrogen oxides $\left(\mathrm{NO}_{\mathrm{x}}\right)$. Particles with a size less than 10 micrometer are of concern due to the inhalable hazard to humans. The particles with size larger than 10 micrometer in diameter will settle down relatively fast in turbulent air ( 8 foot drop takes 5.8 seconds for $100 \mu \mathrm{m}$ particles and 8.2 minutes for $10 \mu \mathrm{m}$ particles) [2]. In addition, according to Environmental Protection Agency of United States (EPA), particles are further categorized into two groups: inhalable coarse particles and fine particles based on their sizes. Larger than 2.5 micrometers and less than 10 micrometers in diameter particles are called inhalable coarse particles that are mostly seen in industries and streets. Fine particles are particles with diameter equal to or smaller than 2.5 micrometers such as smoke and haze. [3, 4]

\subsection{Present Situation}

Cities with manufacturing facilities found that air quality has been greatly weakened compared to years ago. Particulate matter standards were set according by countries showing the importance of role of particulate matter pollution.

PM standard charts are often written in terms of mass concentration of particle per unit volume. A chart has several level (5 to 6 levels) indicating the quality of air from good to bad, and each level has a range indicating particle mass concentration for this category. PM10 concentration usually includes PM2.5 readings. Table 1 is an example of air quality standard published by Environment Protection Agency (EPA) of United States. In the chart, an unhealthy PM10 reading ranges $255 \mu \mathrm{g} / \mathrm{m}^{3}$ to $354 \mu \mathrm{g} / \mathrm{m}^{3}$ and $65.5 \mu \mathrm{g} / \mathrm{m}^{3}$ to $150.4 \mu \mathrm{g} / \mathrm{m}^{3}$ for PM2.5. 
Table 1 Air Quality Standard Breakpoint by EPA [12]

\begin{tabular}{|c|c|c|c|c|c|c|c|c|}
\hline Category & $A Q I$ & $\begin{array}{l}\mathrm{O}_{3} \text { (ppm) } \\
\text { 8-hour }\end{array}$ & $\begin{array}{c}\mathrm{O}_{3} \text { (ppm) } \\
\text { 1-hour }\end{array}$ & $\begin{array}{l}\mathrm{PM}_{25} \\
\left(\mu \mathrm{g} / \mathrm{m}^{3}\right)\end{array}$ & $\begin{array}{c}\mathrm{PM}_{10} \\
\left(\mu \mathrm{g} / \mathrm{m}^{3}\right)\end{array}$ & $\mathrm{CO}$ (ppm) & $\mathrm{SO}_{2}(\mathrm{ppm})$ & $\mathrm{NO}_{2}(\mathrm{ppm})$ \\
\hline Good & $0-50$ & $0.000-0.064$ & (2) & $0.0 \cdot 15.4$ & $0-54$ & $0.0-4.4$ & $0.000-0.034$ & (3) \\
\hline Moderate & $51-100$ & $0.065-0.084$ & (2) & $15.5-40.4$ & $55-154$ & $4.5-9.4$ & $0.035-0.144$ & $\left({ }^{3}\right)$ \\
\hline $\begin{array}{l}\text { Unhealthy for } \\
\text { Sensitive Groups }\end{array}$ & $101-150$ & $0.085-0.104$ & $0.125-0.164$ & $40.5-65.4$ & $155-254$ & $9.5-12.4$ & $0.145-0.224$ & (3) \\
\hline Unhealthy & $151-200$ & $0.105-0.124$ & $0.165-0.204$ & $65.5-150.4$ & $255-354$ & $12.5-15.4$ & $0.225-0.304$ & (3) \\
\hline Very unhealthy & $201-300$ & $0.125-0.374$ & $0.205-0.404$ & $150.5-250.4$ & $355-424$ & $15.5-30.4$ & $0.305-0.604$ & $0.65-1.24$ \\
\hline Hazardous & $\begin{array}{l}301-400 \\
401-500\end{array}$ & $\begin{array}{l}(1) \\
(1)\end{array}$ & $\begin{array}{l}0.405-0.504 \\
0.505-0.604\end{array}$ & $\begin{array}{l}250.5-350.4 \\
350.5-500.4\end{array}$ & $\begin{array}{l}425-504 \\
505-604\end{array}$ & $\begin{array}{l}30.5-40.4 \\
40.5-50.4\end{array}$ & $\begin{array}{l}0.605-0.804 \\
0.805-1.004\end{array}$ & $\begin{array}{l}1.25-1.64 \\
1.65-2.04\end{array}$ \\
\hline
\end{tabular}

1. No health effects information for these levels-use 1-hour concentrations.

2. 1-hour concentrations provided for areas where the AQI is based on 1-hour values might be more cautionary.

3. $\mathrm{NO}_{2}$ has no short-term standard but does have a short-term "alert" level.

Based on the worldwide annual PM10 monitoring record 2008-2014 found in the World health Organization's (WHO) database, the worst air pollution recorded in Peshwar, Pakistan having $540 \mu \mathrm{g} / \mathrm{m}^{3}$ in 2010 while Lake Havasu City-Kingman, AZ, USA has the best air quality in $2012\left(6 \mu \mathrm{g} / \mathrm{m}^{3}\right)$. There are 1453 cities (out of 1524) have particulate matter concentration below $120 \mu \mathrm{g} / \mathrm{m}^{3}$. The other 70 cities where mostly located in Asia and have PM records that exceeded $120 \mu \mathrm{g} / \mathrm{m}^{3}$.

\subsection{PM Monitoring Unit Integrated with a Cellphone}

Currently, there are few portable devices capable of aerosol concentration detection. One can acquire PM reading only from environment facilities or professional monitors. To most people, it is hard to know the air quality of their surrounding and even harder if it's in a closed environment.

In this sense, a device that can do real-time monitoring in any place at any time of a day could help a user to understand the air quality level much more intuitively. Cellphones have become a device that many people cannot "live" without, and it also is a highly integrated device including many kinds of sensors. If an aerosol concentration sensor was able to be integrated with a cellphone but takes little or no extra room, then real time monitoring would be achieved. 


\subsection{Objective}

The object of this thesis is to develop a device that measuring aerosol mass concentration of PM10. The design must have the capability to detect the heaviest PM

pollution record in the history $\left(540 \mu \mathrm{g} / \mathrm{m}^{3}\right)$. Sensing range will then be divided into 10 levels indicating the PM concentration level from good to bad. Physical dimension of the device must have a potential to integrate into a regular cellphone.

\subsubsection{Sub-objective}

1) Choose a PM detection principle that is of low cost, small size, and low power consumption with sensitivity and accuracy enough to satisfy the requirements

2) Conduct the calculation regarding to working principle

3) Select or design optics, fluid unit, and corresponding mechanism for assembly

4) Develop a procedure used for testing and experimental study 


\section{Chapter 2 Technology Review}

There are various physical phenomenon that can be adopted to measure air pollution levels in terms of detecting the concentration of particulate matters. Multiple methods have been reviewed and summarized in this section.

\subsection{Ringelmann Chart Method}

The Ringelmann chart was developed in the late 1800s and adopted by U.S Bureau of Mines in the early 1900s extensively for improving coal burning efficiency and to control emission. On the chart, there are four black grids at different darkness on a white background for measuring the darkness of smoke. The user needs to match the shades of smoke with one of the shades appearing on the chart at a distance of 50 feet from the smoke. Emission of coal can then be classified and fuel-to-air ratio can be adjusted accordingly. [5]
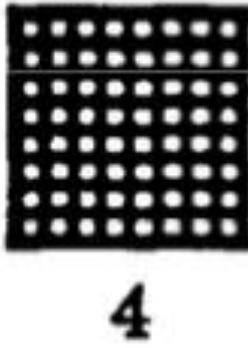
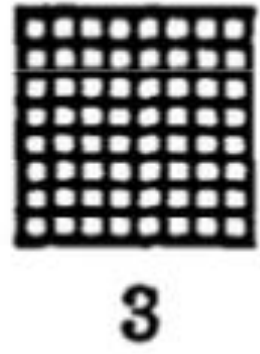

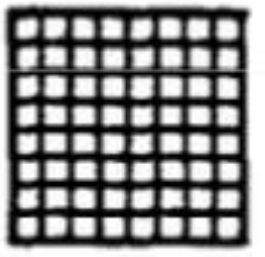

2

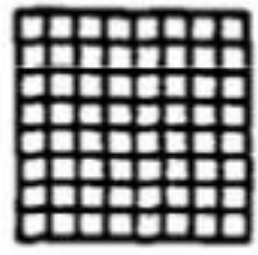

1

Figure 1 Ringelmann Chart [5]

\subsection{Gravimeter Sampler}

Gravimeter sampler is one of the wildly used devices on measuring airborne particulate matter concentration. Because of its high reliability and accuracy, this method became an important regulatory monitoring systems [6].

A pre-weighted filter is used to collect dust over a time period (usually twentyfour hours). The filter is then weighted again, and the mass of collected dust will be determined by the difference of two readings (pre-weight reading and post-weight reading). Dust concentration is mass divided by the volume of drawn air. Since a filter 
has no functionality of dust size selection, dust with desired size has to be preselected beforehand, where cyclonic separation takes place [6].

\subsubsection{Cyclone Separation}

Cyclonic separation is achieved based on centrifugal force. Air and dust flow from a tangential feed into a cylindrical vessel and move in a helix pattern. Since dust particles are much heavier than air, they have much more inertia. This decides heavy particles cannot follow air in a tight cylinder and results in it falling out at the bottom opening of the device after being separated from air. [7]

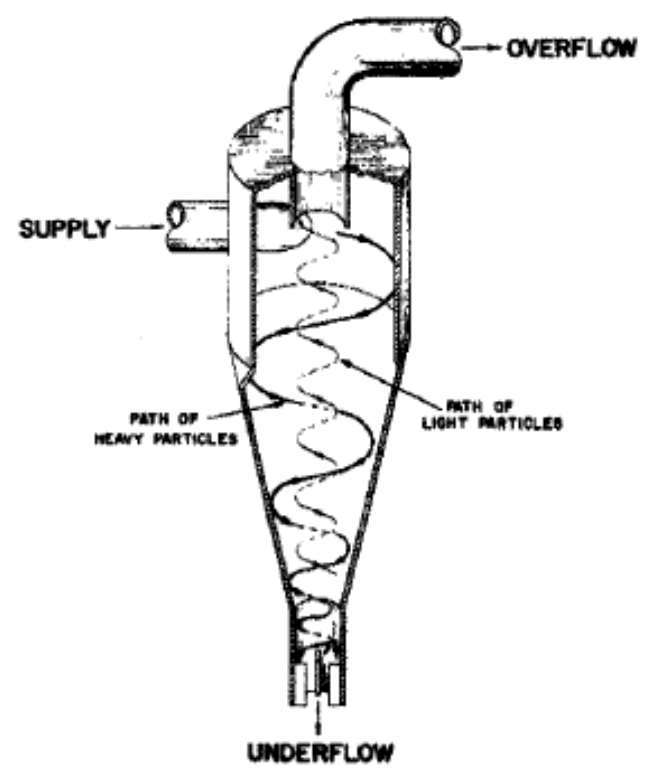

Figure 2 A Typical Cyclonic Separation Design [7]

The volume of air flowing into the system is a key character that decides particle concentration. Thanks to known air flow rate and sampling time, the multiplication of these two gives the air volume. The division of weight and volume of air drawn discloses average dust concentration. The advantage of this method is accuracy. The amount of dust mass can be preciously measured. The collected dust gives the possibility to qualitatively analysis and the concentration to each interesting component as well as their other physical and chemistry properties. However, this method is more time consuming, as it has a longer period for sampling time, which makes real-time monitoring impossible. 
Also, human interact is unavoidable: the filter has to be weighted before and after the collection.

\subsection{Thin-Film Bulk Acoustic Resonator (FBAR)}

The main component of this method is a resonator that oscillates at a known frequency. The resonator also acts as a sensor that measures the frequency change due to add-on mass of dust $[8,9]$. According to Sauerbrey equation:

$$
\frac{\Delta \mathbf{f}}{\mathbf{f}}=\frac{\Delta \mathbf{m}^{\prime}}{\mathbf{m}^{\prime}}
$$

where $\mathbf{m}^{\prime}=$ mass per unit area of the device, $\Delta \mathrm{m}^{\prime}=$ added mass per unit area of dust, $\mathbf{f}=$ frequency, and $\Delta \mathrm{f}=$ frequency change [9], additional mass is able to be calculated with known surface area of the resonator.

Thermophoresis precipitator is utilized to deposited small particles onto the resonator. Particles are moving against temperature gradient, i.e. moving from high temperature area to low temperature area, because of the collision with different kinetic energy gas molecules [9].

The advantage of this design is its size and power consumption. A drawback may be the requirement of air flow which is produced by a fan. A fan requires a relatively big physical dimension; hence, the space occupied by the entire system will be large. In addition, the cleaning of the dust deposited on the resonator may become difficult and requires human interaction. The design cannot distinguish different sizes of particles. With the attachment of a ultra-violet or infrared light, more physical and chemical characteristics of dust can be studied at the same of concentration measurement. 


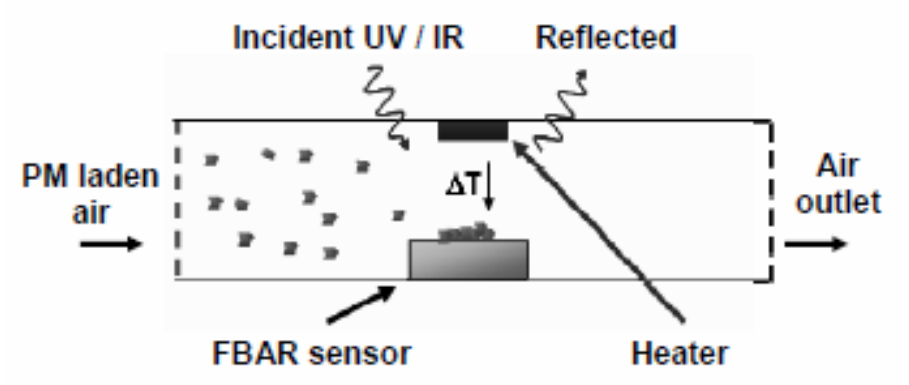

Figure 3 Schematic of FBAR System (Incident UV/IR is used for discriminating the nature of particles) [9]

\subsection{Tapered Element Oscillating Microbalances}

Tapered Element Oscillating Microbalances (TEOM) are developed from a gravimetric system. The tapered element contains a hollow glass tube that is free to vibrate at its natural frequency. There is a filter cartridge mounted on the tip of the glass tube and is used to collect dust particles. Any additional weight depositing on top of the filter changes the glass tube oscillating frequency. An electric circuit will detect frequency change and therefore the mass gain rate will be calculated from the difference of frequency magnitudes. The system reset its oscillation frequency of vibrating glass tube back to its natural frequency prior to the next measurement is ready. Further, dust concentration can be calculated by dividing the mass rate by the air flow rate. [6]

A cyclone head may be used to separate desired size of dust from un-interesting size particles. This could be the limitation of TEOM on reducing the size of the entire system.

TEOM provides a continuous output of the particle concentration measurement. Air flow rate has to be uniform over the monitoring period in order to give accurate measurement. Obviously, the advantage of TEOM could be providing real-time and continuous monitoring. The result will be the mass concentration (i.e. mass per unit of volume) that is more accurate than number concentration (i.e. number of particle per unit of volume). 


\subsection{Light Absorption Method}

Light does not only scatter when it comes to a small particle: some of the light could also be absorbed by the particle. Light absorption is based on absorbing light energy to indirectly make the measurement of dust concentration.

The entire setup of this measurement device contains an infrared LED, two photodiodes and a dust filter inside of an optic chamber which has an air inlet and outlet. A new and clean filter is inserted into the position. Photodiode 1 and 2 receive two readings while infrared light is emitting. The difference of photodiode 1 and 2 readings is calculated and recorded. Air pump is then turned on and air containing dust flows into the optic chamber from air inlet. Filter separates dust from air and collects it over the sampling time. Air pump will be turned off once the sampling time is over. Photodiode 1 and 2 will take another two readings. The difference between the two new readings by photodiode 1 and 2 will be calculated and recorded. The first and second differences are representing mass of clean filter and total mass of clean filter plus dust. [10]

Both photodiodes measure the intensity of light detected rather than the mass of filter or dust. However, by knowing the light intensity and corresponding dust mass, the relationship between light attenuation and mass of dust could be found after several experiments. Using this relationship, the mass of dust will be determined once the amount of light attenuation is known.

This method is not capable of measuring the size distribution of dust. Therefore, in order to measure dust solely within specific ranges of size, a preselecting process have to be done. Cyclone separation device could be one of the options. Detailed explanation of cyclone separation working principle can be seen in gravimeter sampler method.

The advantage of this method is it's easy to understand and to use. The downsides of this method are several. The light attenuation may be negligible to be detected in a short distance. Reselection according to dust physical size may be limit size minimization of entire system. Filter replacement needs human interaction, and real-time and/or continuous measurement is impossible to be achieved. Sampling time may have to be long enough to collect sufficient dust layer to make the measurement. 


\subsection{Beta Attenuation Monitor (BAM)}

This method is an indirect method, which is measuring the reduction of beta source radiation. A thicker dust layer increases the attenuation speed of beta source which means less beta count will be received by the detector. The system contains beta source (radiation generator), beta detector (radiation detector), and filter. ${ }^{14} \mathrm{C}$ and ${ }^{85} \mathrm{Kr}$ are good candidates to be chosen as beta sources.

First, an unused filter is spotted between beta source and beta detector, and count of beta radiation is measured by beta detector and then recoded. The filter is moved to in front of air inlet where dust and air are drawn into the system. The filter is dense enough to remove the dust floating in the air while air is free to pass through. Over a period of time, enough sampling dust is collected. The filter is moved back to in between the beta source and detector. The second count of beta radiation is recorded. Using the two different counts, the amount of attenuation of beta radiation is determined, which also reveals the mass of dust collected on the filter. With known air entering rate and sampling time, the mass concentration of airborne particulate matter can be easily calculated. [6]

BAM can be very accurate and provide continuous operation, which purposely designed mechanism. Since the dust is collected on filter, size distribution analysis and qualitative analysis are possible to be processed with associated devices. BAM contains enough radioactive source to be fatal so that licenses are required to all operators. Due to BAM its working principle, BAM usually comes big in size and heavy in weight. In addition, the filter has to be changed every time after each measurement. Relatively long sampling period of time is required, which also reveals significant power consumption. Temperature, humidity, and pressure are other influences to the measuring result [6].

\subsection{Charge Measurement}

Dust is believed to contain charge. Induced current will be detected due to existing of dust, and the magnitude of the current has linear relationship with the amount of charge [11]. Higher the magnitude means more charge existing, and more charge means more dust. In the specific volume of area; however, more dust does not necessarily correspond to higher concentration of dust. 
Air flow rate plays an important role in this method. At a constant concentration of dust and the air flow speed is ranged between $8 \mathrm{~m} / \mathrm{s}$ to $18 \mathrm{~m} / \mathrm{s}$, higher air current speed induces higher magnitude of current [11]. Therefore, it is important to maintain a constant air flow speed during the measurement.

\subsection{Image Method}

Image method may be the easiest method to determine the dust concentration level. An image of dust (Figure 5) is captured in a dark room in which only a 2-D light surface is produced by directed halogen lights while everywhere else is kept dark [12]. Then the image processing has to be done and a contour plot (Figure 6) is generated for the further analysis. This method is good for qualitative monitoring system [6] due to its inaccuracy during the image processing.

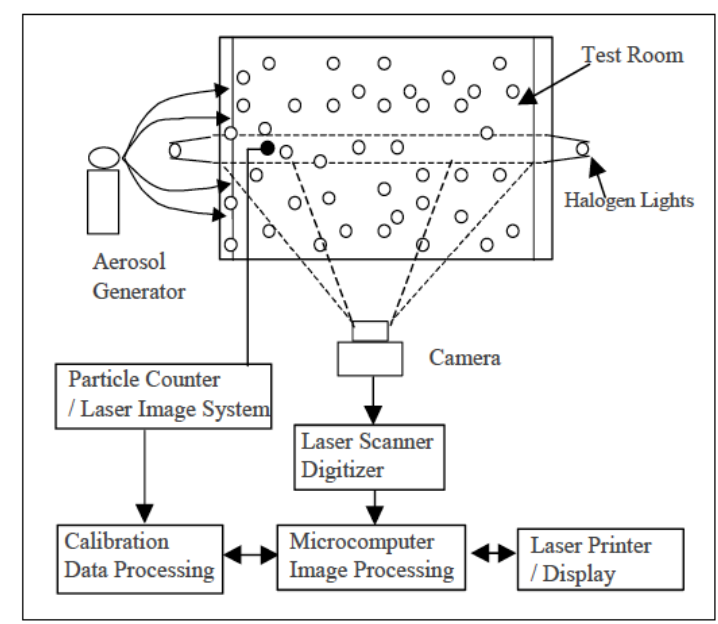

Figure 4 Schematic of Image Method Setup [12] 


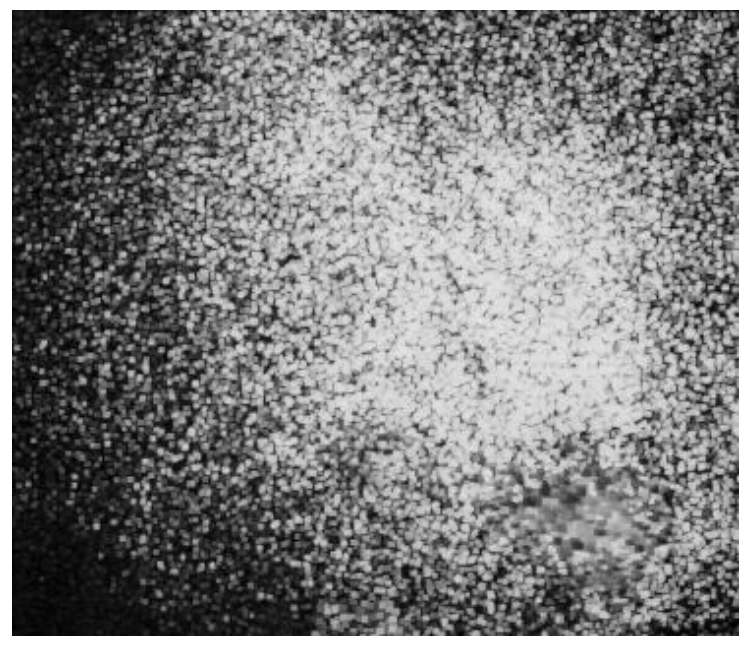

Figure 5 A Taken Image of Particles [12]

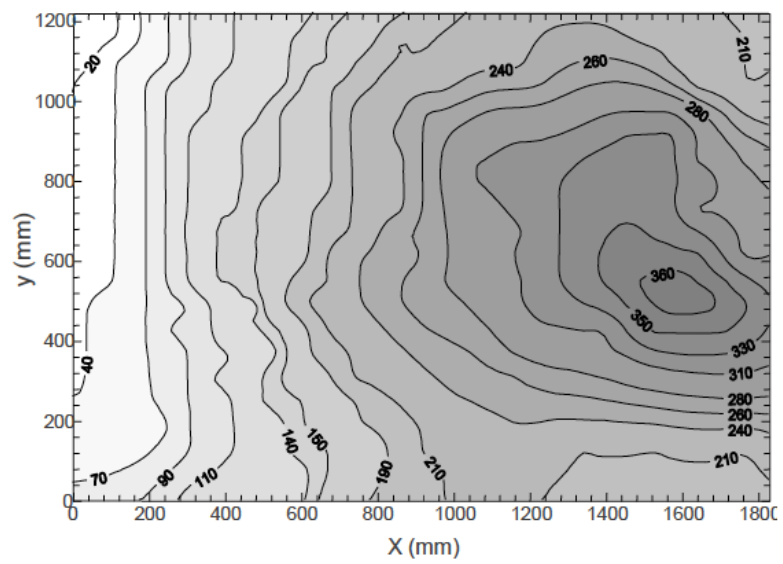

Figure 6 Contour Plot of Particle Concentration by Image Process [12]

\subsection{Particle Counting}

Particle counting method is one of the method using light scattering phenomenon. Incident light is scattered by a particle and the scattered light intensity is received. Received signal is then be analyzed to determine size of the corresponding particle. Over a period of time, size of the particles passed across detection region is collected and the number of the particles within desired size range can be counted. Known Volumatic air flow is used to determine the number concentration of particles. This method can provide accurate result in terms of number concentration. However, a period of sampling time is required. 


\subsection{Comparison of all Methods}

The Ringelmann Chart method might be the least accurate method among all. Gravimeter sampler requires a filter and takes long sampling time. FBAR may generate high temperature to produce thermophoresis, and it consumes power significantly due to high temperature. TEOM may hard to shrink size because of its architecture. Light absorption needs a high power light source which may drain a battery. BAM is big in size and also requires long sampling time and license to operator because of radiation. Charge measurement needs high flow rate and long sampling time. Image method is suitable for qualitative analysis rather than quantitative analysis. Although particle counting gives accurate result, a period of sample is unavoidable that leads to higher power usage comparing to instantaneous measurement. 


\section{Chapter 3 Virtual Impactor Based Light Scattering Sensing}

\section{Unit}

\subsection{Working Principle}

The entire design can be divided into three systems: airflow system, particle separation system and particle mass concentration measurement system. The air flow provided by a suction fan to produce pressure at two ends of the ventilation duct, producing air flows from higher pressure duct to lower pressure duct. Once particles are drawn into the ventilation duct, particles will be going through particle separation system. A virtual impactor is used here to select desired size particles. Large particles will exit the system to atmosphere directly without being measured. Small particles will flow through the major flow where the measuring unit located, and the concentration is about to be detected. The measuring unit includes a laser module and a photodiode.

Figure 7 shows the work flow of virtual impactor based light scattering sensing unit.

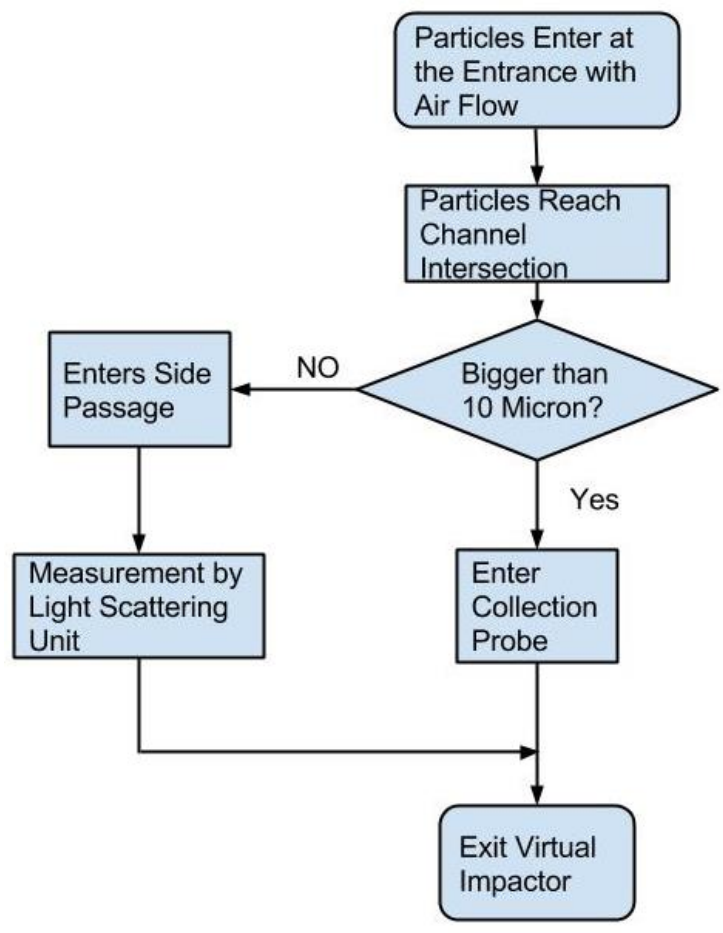

Figure 7 Work Flow of the PM10 Monitoring Unit 


\subsection{Advantage of a Virtual Impactor}

The virtual impactor is unlike a normal impactor which uses an impact plate to collect large particles and has a chance to cause overload [13]. Virtual impactors provide convenience during lifetime to customers: there is no cleaning and replacement process needed.

\subsection{Consideration of the Design}

The sensor has to be sense airborne particulate matter with and without daylight. Ambient light intensity should not be one of the major affects to the measurement. The readings could be influenced by temperature, pressure and/or humidity in a reasonable range. Human interaction during the measurement will be excluded. The device will have potential to imbed in a regular smart phone, which means size is essential. The height of the system must be less than or equal to the thickness of a smart phone. Length and width of the entire system have to be smaller than the length and width of the cell phone, respectively. Short sampling time has benefits not only as fast a response device, but also consume less power usage. A smart phone is unlike an old fashioned telephone with a solid line, it does not draw electricity from power outlet. The battery limits the usage of power to all features on a cell phone, which requires the device power consumption to be efficient. A normal cell phone battery capacity ranges from $1500 \mathrm{mAh}$ to $3000 \mathrm{mAh}$. Last but not least, the sensor must be user friendly and have no harm to all users.

\subsection{Integration with a Cellphone}

The purpose of the device is to be integrated with a regular cellphone. A strict mechanical challenge is the overall dimension of the device. There are two possible solutions for this design to integrate with a cellphone, which are either integration internally as a component during manufacturing and assembly process or integration as a cellphone accessory. 


\subsubsection{Integration with a Cell Phone}

3.4.1.1 As an Internal Component

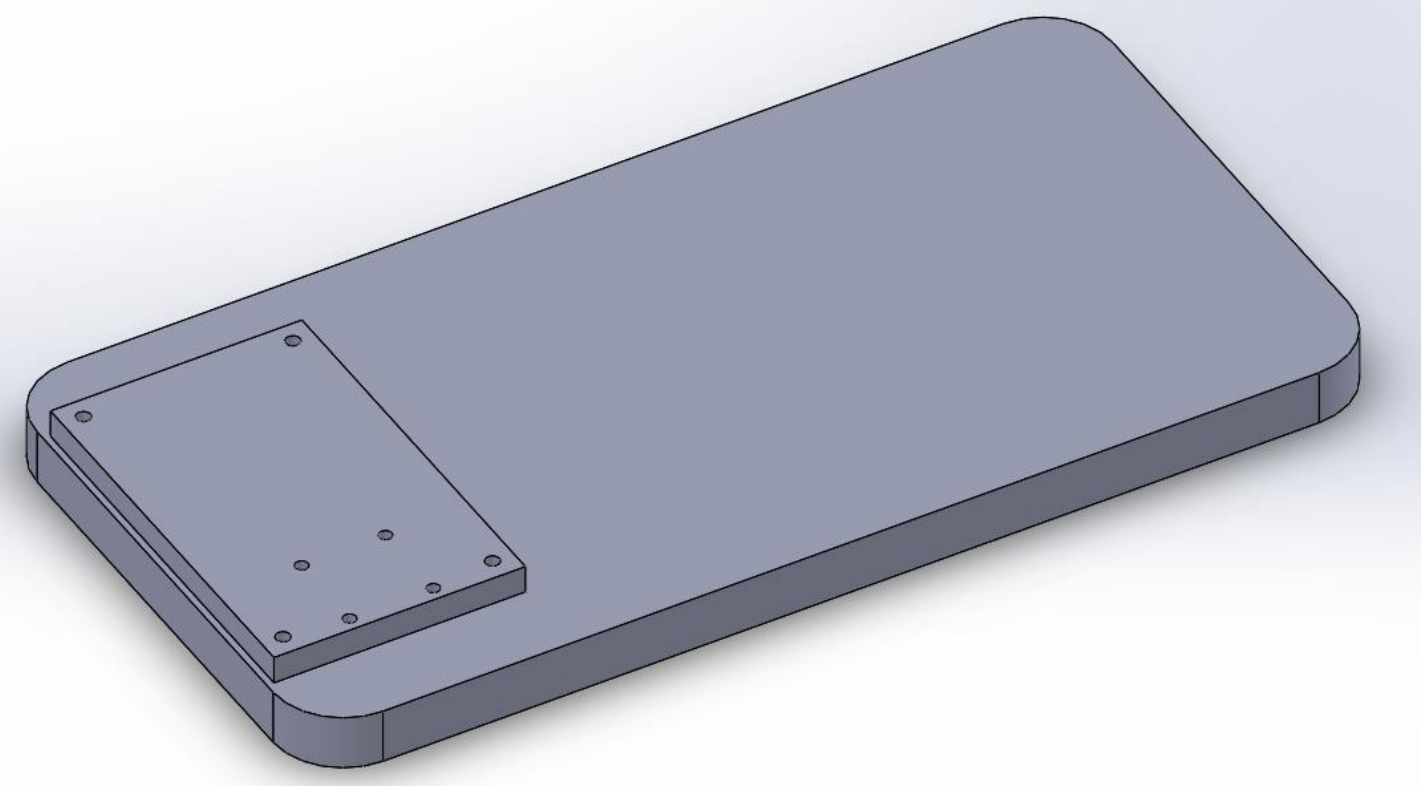

Figure 8 Schematic of Integration: as an Internal Component (Back Lid Removed)

Figure 8 shows an idea of design integrated in a cellphone as an internal component. The lower part of phone is manufactured $4 \mathrm{~mm}$ thicker. The circuits of laser, photodiode and fan are connecting to phone circuitry.

\subsubsection{As an Accessory}

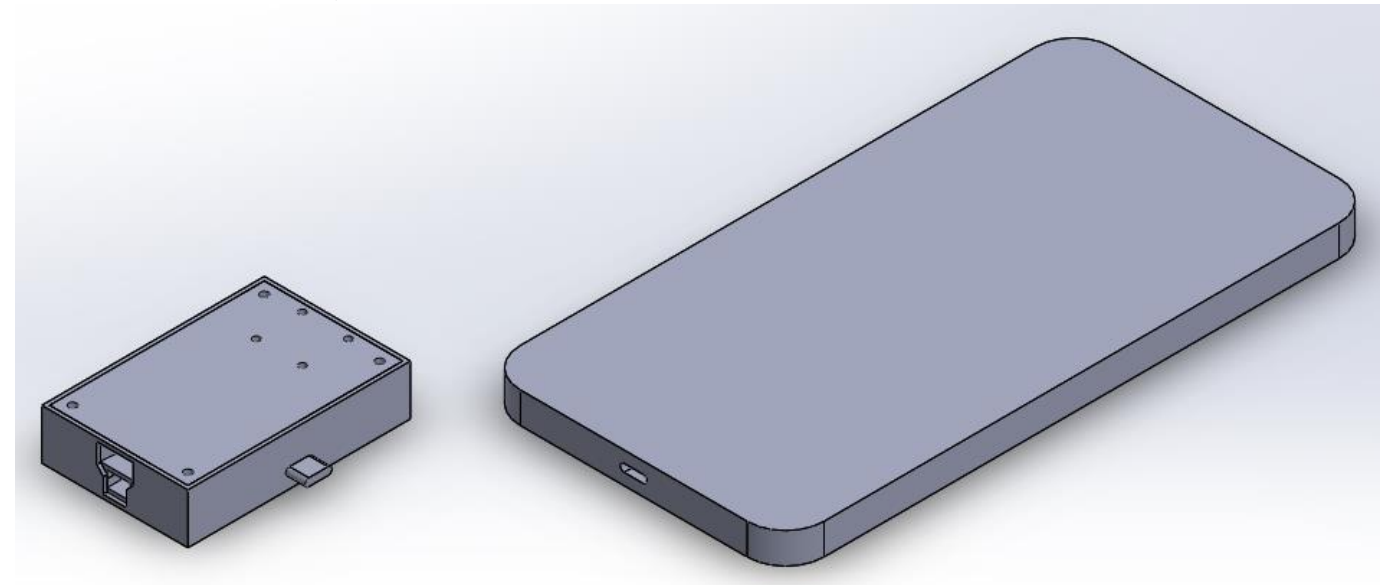

Figure 9 Schematic of Integration: as an Accessary

A USB connecter provides data transfer and power supply. An associated application will be used to display measurement result and view history records. 


\subsubsection{Current Design}

Overall dimension of current design is $5.6 \mathrm{~cm}$ (Length) $* 3.6 \mathrm{~cm}$ (Weight) $* 1.2 \mathrm{~cm}$ (Height) including a 3D printed structure (base and cover, seen in Appendix), a rotary fan, a laser module and a photodiode. The thickness of air channel wall is $1 \mathrm{~mm}$, which is limited by the current 3D printing technology. The design includes laser diode and photodiode mounts. Associated holes for fan mount are considered. Cover has an inner air channel where particle separation happens. When the cover and base are assembled, they form fluid two fluid channels: one for inlet air flow and the other one is for particles in two major flow gathering and then exiting. Moreover, screws and nuts fasten two parts of the air channel.

\subsubsection{Fan}

The selection of fluid unit is based on three physical quantities: dimension, power and flow rate. Since steady flow rate gives fast and accurate measurement, a rotary fan is determined. As a prerequisite, to ensure enough flow rate is generated, dimension and power consumption are following the lowest among all searched products.

\subsubsection{Photodiode}

The Detectivity of a photodiode reveals the weakest light intensity that can be detected. The most expression for detectivity used across different manufacturers is in terms of Noise Equivalent Power (NEP), which originally describe the incident light power that gives a signal-to-noise ratio $(\mathrm{S} / \mathrm{R})$ of 1 at an output bandwidth frequency of $1 \mathrm{~Hz}$ [14]. Lower NEP value is better since it corresponds to a lower noise floor or a weaker light signal can be distinguished from noise [14].

In order to calculate the lowest amount of light intensity can be detected, the corresponding NEP at given wavelength $(\operatorname{NEP}(\lambda))$ has to be known by using equation (3.1) [14]:

$$
\operatorname{NEP}(\lambda)=\mathrm{NEP}_{\text {min }} \times \frac{R_{\text {max }}}{R(\lambda)}
$$

where $\mathrm{NEP}_{\min }$ is the NEP happens at $\mathrm{R}_{\max }\left(=1.6 \mathrm{E}-15 \mathrm{~W} / \mathrm{Hz}^{0.5}\right), \mathrm{R}_{\max }$ is the highest photosensitivity $(0.36 \mathrm{~A} / \mathrm{W}$ happens at $720 \mathrm{~nm}), \mathrm{R}(\lambda)$ is the photosentivity at selected wavelength of $650 \mathrm{~nm}(=0.35 \mathrm{~A} / \mathrm{W}$, approximately according to Figure 10$)$ (taken from 
datasheet of Hamamatsu, seen in Appendix). Calculated NEP $(\lambda)$ is $1.65 \mathrm{E}-15 \mathrm{~W} / \mathrm{Hz}^{0.5}$. The lowest amount of light $\left(\mathrm{P}_{\min }\right)$ that can be detected by this photodiode is computed by the expression [14]:

$$
\mathbf{P}_{\min }=\mathbf{N E P}(\lambda) \times \sqrt{\mathbf{B W}}
$$

where BW is measurement bandwidth (in this thesis, measurement bandwidth of $10 \mathrm{kHz}$ is taken). Therefore, $\mathrm{P}_{\min }$ is $1.65 \mathrm{E}-10 \mathrm{~W}(=1.65 \mathrm{E}-7 \mathrm{~mW})$.

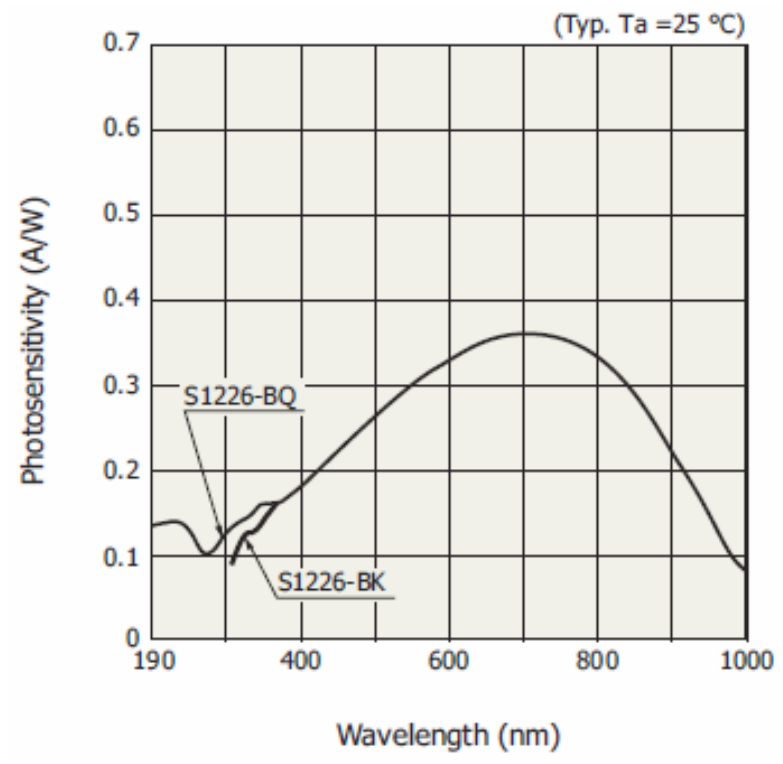

Figure 10 Photodiode Spectral Response Diagram [15]

\subsubsection{Laser Module}

Two limitations are present when choosing the laser module: laser output power and light wavelength. Laser output power is essential when considering light intensity has to be strong enough to be cover entire desired detection range and safety requirement to people who deal with it. Wavelength also determines parameter of design on scattering detection. Based on the selection of photodiode, the laser module (product number: ML51G-650) was chosen from world star tech (specification in Appendix). Output power of $1 \mathrm{~mW}$ is classified as Class II, which reduces the risk of eye damage in misusing or in accident. Wavelength of $650 \mathrm{~nm}$ makes calibration process easier due to visibility. Specification of the laser module can be viewed in Appendix. 


\subsubsection{Improvement}

One of the most important improvements will be on physical dimension. First of all, the air channel is preferably not 3D printed for achieving minimum thickness while remaining rigid. The main body of cellphone may be redesigned in such a way that part of it is an air channel as well as the mechanism where laser and photodiode mount. Back cover of the phone is also being the top lid of the air channel and therefore entire thickness would be decreased by at least $2 \mathrm{~mm}$, which is equal to the wall thickness of current design. Secondly, the laser module would be replaced by laser diode for further overall dimension reduction. 


\section{Chapter 4 Light Scattering}

In this chapter, the physics behind light scattering will be explained first followed by introducing two main light scattering theory: Rayleigh scattering and Mie scattering. Equations of Mie scattering will be detailed explained and derived in the form for calculating particle mass concentration. Sensitivity and sensing capability of the design with selected components are conducted through MatLab programming.

\subsection{Light Scattering}

Light is an electromagnetic wave with its electrical wave perpendicular to its magnetic wave while both waves are perpendicular to the direction of propagation. The electron orbits in molecules of a particle are disturbed and oscillate at the same frequency as the incident light when it is incident on a particle. The oscillation causes periodically separation of charges contained by the molecules; this phenomenon is called induced dipole moment and observed as redirection of light, or simply light scattering. [16, 17]

Figure 11 shows the redirection of incident electromagnetic wave by a small particle by induced dipole moment.

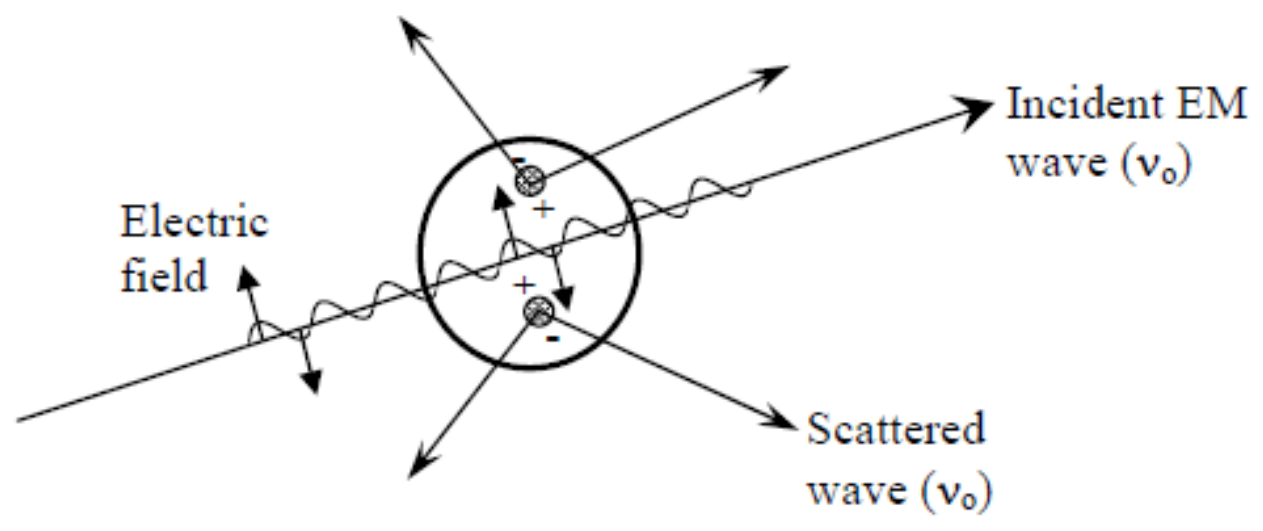

Figure 11 Light Scattering Due to Incident EM Wave [18] 
Incident light after scattering propagates in all direction of three dimensional space. Figure 12 qualitatively visualized the phenomenon of light scattering by single particle on a two dimensional surface.

Elastic scattering and inelastic scattering are two types of light scattering theory. Elastic scattering is employed when the scattered light frequency remains the same as incident light frequency, such as Rayleigh scattering. When scattered light frequency changes during light scattering and results in scattered light having different frequency as incident light frequency, an inelastic scattering has happened, such as Raman scattering. [19]

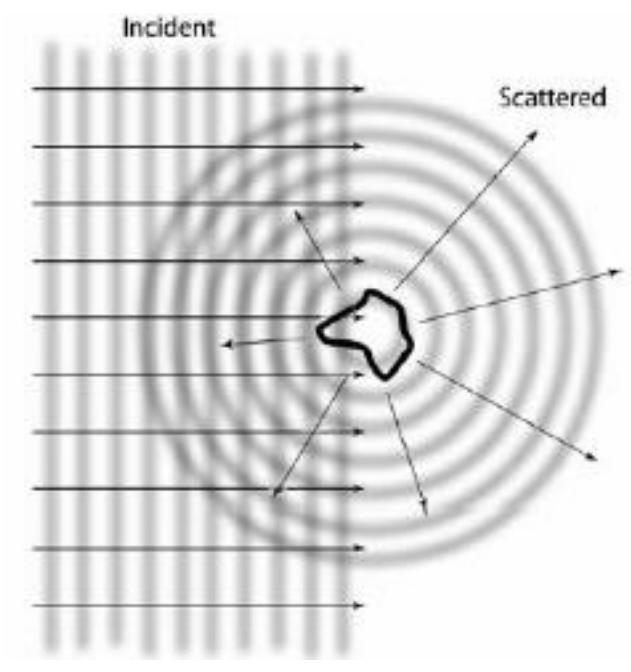

Figure 12 Visualization of Light Scattering by a Small Particle [20]

The direction of scattered light is depending on the wavelength of incident light and particle size. According to Figure 13 and Hu [19]: to small particles (Figure 13 case A), backward scattering tends to in equilibrium with forward scattering; to big particles (Figure 13 case B and C), there is more light scattered forwardly than in backward direction. 


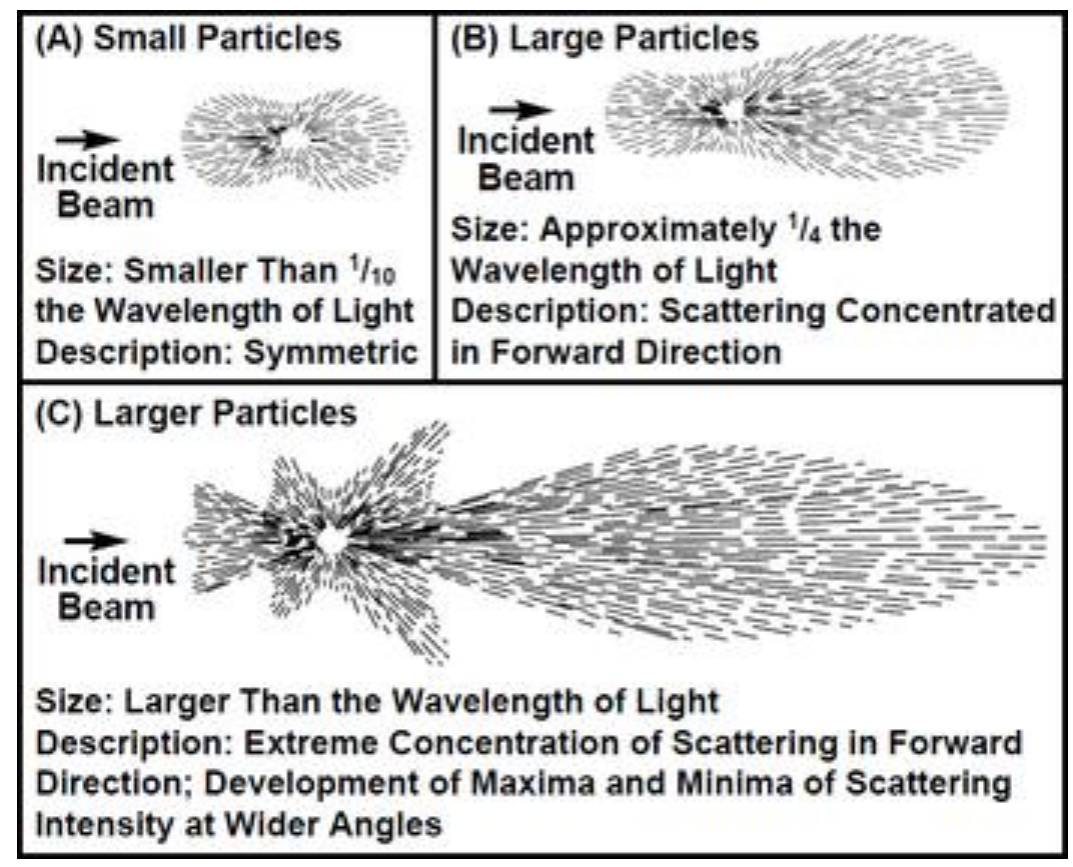

Figure 13 Scattered Light Patten by Different Size of Particles [21]

Consider there is solely particle in a space and light is scattered by the particle, this type of scattering is categorized in primary scattering when light scatters only once. However, in most situations, more than one particle presents in a studied volume of space. It is highly possible that scattered light is scattered again by other particles. This phenomenon is called secondary scattering or multiple scattering if more times of scattering happens. Multiple scattering has little affect to the extinction of light but changes the distribution of scattered light intensity [19].

Light scattering technique is one of the most popular methods that wildly used in the field of optics. There are two fundamental theories regarding to small particles scattering: Rayleigh scattering theory and Mie scattering theory.

\subsection{Rayleigh Scattering}

British physicist Lord Rayleigh (John William Strutt) developed Rayleigh scattering theory and first explained why the sky is blue and sunset is red. This theory applies to small spherical dielectric particles [18]. Mie scattering theory is believed to analysis the most spherical particle scattering system (including Rayleigh scattering) without size limitation [18]. Nevertheless, Rayleigh scattering is employed because of its 
simplicity of formulation. The determination of Rayleigh scattering is when $\mathrm{x}<<1$ and $|\mathrm{m}| \mathrm{x} \ll<1$, where $\alpha$ is called size parameter which calculated in the following equation [18]:

$$
x=\frac{2 \pi a}{\lambda}
$$

where $\mathrm{a}$ is the radius of spherical particle, and $\lambda$ is the relative scattering wavelength which defined as

$$
\lambda=\frac{\lambda_{0}}{\mathbf{m}_{0}}
$$

where $\lambda_{0}$ is the incident light wavelength, and $m_{0}$ is refraction index of the surrounding media. Finally, $\mathrm{m}$ is the refractive index of the particle and represented in complex notation

$$
\mathbf{m}=\mathbf{n}-\mathbf{i k}
$$

where $\mathrm{n}$ is the real part which denotes the refraction of the light and $\mathrm{k}$ is the imaginary part which is a parameter related to absorption. Absorption coefficient of a material is expressed as

$$
\text { Absorption Coefficient }=\frac{4 \pi k}{\lambda}
$$

The value of $\mathrm{k}$ is usually a nonzero number, then the magnitude of refractive index as needed for Rayleigh criteria could be calculated by using the same technique as calculating the magnitude of a complex number:

$$
|\mathbf{m}|=\sqrt{\mathbf{n}^{2}+\mathbf{k}^{2}}
$$

Intensity of scattered light varies at different combination of reception angle and distance from the scattered particle to reception surface (often a photodiode is used to receive and detect level of scattered light intensity). The light intensity I scattered by particle of diameter $d$ received at distance $r$ and angle $\theta$ is determined with the expression [19]:

$$
I=\frac{d^{6} \pi^{4}}{8 \lambda^{4} r^{2}}\left(\frac{m^{2}-1}{m^{2}+2}\right)^{2}\left(1+\cos ^{2} \theta\right) I_{0}
$$


where $\lambda$ is the incident light wavelength, $m$ is the refractive index of surrounding medium and $\mathrm{I}_{0}$ is the incident light intensity. The above equation can also be written in terms of the molecular polarizability $\alpha$ :

$$
I=I_{0} \frac{8 \pi^{4} \alpha^{2}}{\lambda^{4} r^{2}}\left(1+\cos ^{2} \theta\right)
$$

$\alpha$ is given by Lorentz-Lorenz formula:

$$
\alpha=\frac{3}{4 \pi N}\left(\frac{m^{2}-1}{m^{2}+2}\right)^{2}
$$

where $\mathrm{N}$ is the number of particles per unit volume. For a single molecule scattering, $\mathrm{N}=1$.

Considering the interest of this thesis, as stated previously, particle size is up to $10 \mu \mathrm{m}$, which is significantly greater than molecules (whose size is in the order of one hundredth of a micrometer). Therefore, Rayleigh scattering will not be applicable due to the in-accuracy of the measurement.

\subsection{Mie Scattering}

Mie Theory is often called Mie-Debye Theory or Lorentz-Mie theory. Two important assumptions are made: the particle is a sphere and the particle is homogeneous. One advantage of using Mie scattering theory, as mentioned before, is it is not limited to particle size. Unlike Rayleigh scattering, Mie scattering is able to be used on particle with size smaller than the incident light wavelength as well as large particles. To large particles, Mie equations will be converged to limitation of geometric optics (ray optics) such as reflection, refraction and diffraction $[18,19]$.

\subsubsection{Maxwell Equation}

Since light is an electromagnetic wave and Maxwell equations are used to describe the behavior of electric and magnetic fields. Mie gives an exact solution to Maxwell equations for monochromatic light scattered by a homogeneous sphere. The Maxwell equations contain four rules, Gauss' Law, Gauss Law for Magnetism, Faraday's Law and Ampere's Law, each rule explains the natural of either electric field or magnetic field: 


$$
\begin{aligned}
& \boldsymbol{\nabla} \cdot \mathbf{D}=\boldsymbol{\rho}\left(\text { Gauss' }^{\prime} \text { Law }\right) \\
& \boldsymbol{\nabla} \cdot \mathbf{B}=\mathbf{0} \text { (Gauss' Magnetism Law) } \\
& \nabla \times E=-\frac{\partial B}{\partial t} \quad(\text { Faraday's Law }) \\
& \boldsymbol{\nabla} \times \mathbf{H}=\mathbf{J}+\frac{\partial \mathrm{D}}{\partial \mathrm{t}} \quad(\text { Ampere's Law })^{2}
\end{aligned}
$$

where $\mathrm{D}$ is electric flux density, $\rho$ is amount of charge per unit volume, B is magnetic flux density, $\mathrm{E}$ is electric field, $\mathrm{H}$ is magnetic field, $\mathrm{J}$ is conductivity of material, and $\mathrm{t}$ is time.

The Gauss' Law governs the electric field surrounding electric charges. The formula shows that the divergence of electric flux density equals to electric charge density. Divergence is a vector operator that measures the magnitude of outward flux density at a known point. A positive charge causes flow into a volume as a source adds water into a reservoir while a negative charge acts as a sink to make flow out of a volume as water is drained from a reservoir. Divergence of a magnetic field $\mathrm{H}$ is determined by

$$
\boldsymbol{\nabla} \cdot \mathbf{H}=\frac{\partial H_{x}}{\partial x}+\frac{\partial H_{y}}{\partial y}+\frac{\partial H_{z}}{\partial z}
$$

where $\mathrm{H}_{\mathrm{x}}, \mathrm{H}_{\mathrm{y}}$ and $\mathrm{H}_{\mathrm{z}}$ are magnitude of magnetic field in $\mathrm{x}, \mathrm{y}$ and $\mathrm{z}$ direction, respectively. It shows the rate of change of $\mathrm{H}$ in any direction ( $\mathrm{x}, \mathrm{y}$ or $\mathrm{z}$ direction). More generally, the divergence of an electric field in any specified volume is equal to the net amount of charge in the same volume.

The Gauss' Law for magnetism indicates the divergence of a magnetic flux density is zero, which can be understood as there is no magnetic monopoles existing (magnetic poles, North $\mathrm{N}$ and South $\mathrm{S}$, come in a pair). Magnetic flux density is proportional to magnetic field by

$$
B=\boldsymbol{\mu} \mathbf{H}
$$

where $\mu$ is the permeability of the material. Therefore, the Gauss' Law for magnetism can be rewritten in the form of

$$
\boldsymbol{\nabla} \cdot \mathbf{H}=\mathbf{0}
$$


where $\mathrm{H}$ is magnetic field.

Faraday's Law tells the change of circuit enclosed magnetic flux with respect to time is in equilibrium with the negative induced electromotive force (EMF) in a closed circuit. The formula shows the curl of electric field is equal to the time rate of magnetic flux density change. The curl is vector operator, which measures the rotation of a vector field. The curl of an electric field $\mathrm{E}$ is determined by

$$
\nabla \times E=\left(\frac{\partial E_{z}}{\partial y}-\frac{\partial E_{y}}{\partial z}\right) \hat{x}+\left(\frac{\partial E_{x}}{\partial z}-\frac{\partial E_{z}}{\partial x}\right) \hat{y}+\left(\frac{\partial E_{y}}{\partial x}-\frac{\partial E_{x}}{\partial y}\right) \hat{z}
$$

where $E_{x}, E_{y}$ and $E_{z}$ are magnitude of electric field in $x, y$ and $z$ direction, respectively.

The equation above expresses the result of curl operator, which reveals the rotation of the field in any of the three principal axis ( $\mathrm{x}, \mathrm{y}$ and $\mathrm{z}$ axis). Therefore, Faraday's equation implicates that the electric field changing in time creates magnetic field, and vice versa.

The Ampere's Law shows the magnetic field induced by a known electric current or the current induced by a known magnetic field. Its original form was uncompleted until Maxwell introduced displacement current density term (term $\partial \mathrm{D} / \partial \mathrm{t}$ ). Term $\mathbf{J}$ is called electric current density which is defined as the total flow of charge per time over a cross section of area. The current is the integral of electric current density over surface $S$ where current flows across:

$$
\mathbf{I}=\int_{\mathbf{S}} \mathbf{J} \cdot \mathbf{d S}
$$

In addition, electric current density is related to electric field as:

$$
\mathbf{J}=\boldsymbol{\sigma} \mathbf{E}
$$

where $\sigma$ is the conductivity of the material.

\subsubsection{Mie Solution to Maxwell Equations}

In order to have an answer, Mie had made two important assumptions to simplify the complexity of Maxwell equations: particle is a sphere which has a radius of $\mathrm{d}$ and particle is homogeneous which indicates the refractive index remains the same at a given 
wavelength [19]. Moreover, the term of far-field zone is employed, which means the observation is at a large distance (relative to the size of the particle) away from the scattered particle [22].

The solution to wave equation at distance $\mathrm{R}$ from the scattered particle (sphere) is given as [22]

$$
\left[\begin{array}{l}
E_{1}^{s} \\
E_{r}^{s}
\end{array}\right]=\frac{e^{-i k R+i k z}}{i k R}\left[\begin{array}{ll}
S_{2} & S_{3} \\
S_{4} & S_{1}
\end{array}\right]\left[\begin{array}{l}
E_{l}^{i} \\
E_{r}^{i}
\end{array}\right]
$$

Where $\mathrm{k}$ is the spatial frequency which is calculated as $2 \pi / \lambda, \mathrm{E}_{\mathrm{l}}^{\mathrm{S}}$ and $\mathrm{E}_{\mathrm{r}}^{\mathrm{s}}$ are the parallel and perpendicular components of incident electrical field, respectively, $\left[\begin{array}{ll}S_{2} & S_{3} \\ S_{4} & S_{1}\end{array}\right]$ is the unit-less amplitude scattering matrix and $\mathrm{e}^{\mathrm{i} k z}$ is the incident plane wave. For spheres, $S_{3}$ and $S_{4}$ are zero vectors, then equation (4.19) can be reduced to

$$
\left[\begin{array}{l}
E_{l}^{s} \\
E_{r}^{s}
\end{array}\right]=\frac{e^{-i k R+i k z}}{i k R}\left[\begin{array}{cc}
S_{2} & 0 \\
0 & S_{1}
\end{array}\right]\left[\begin{array}{l}
E_{l}^{i} \\
E_{r}^{i}
\end{array}\right]
$$

Using $\theta$ denote the angle between incident light and scattered light, then the scattering amplitude $\left(S_{1}\right.$ and $\left.S_{2}\right)$ at this angle $\theta$ can be calculated in the given equation set [22]:

$$
\begin{aligned}
& S_{1}(\theta)=\sum_{n=1}^{\infty} \frac{2 n+1}{n(n+1)}\left[a_{n} \pi_{n}(\cos \theta)+b_{n} \tau_{n}(\cos \theta)\right] \\
& S_{2}(\theta)=\sum_{n=1}^{\infty} \frac{2 n+1}{n(n+1)}\left[b_{n} \pi_{n}(\cos \theta)+a_{n} \tau_{n}(\cos \theta)\right]
\end{aligned}
$$

In (4.21) and (4.22), $\pi_{n}$ and $\tau_{n}$ are Mie angular functions expressed in the term of the Legendre polynomials and $\mathbf{a}_{\mathbf{n}}$ and $\mathbf{b}_{\mathbf{n}}$ are called Mie coefficient by [18, 20, 23, 24, 25]:

$$
\begin{gathered}
\pi_{n}(\cos \theta)=\frac{P_{n}^{(1)}(\cos \theta)}{\sin \theta} \\
\tau_{n}(\cos \theta)=\frac{d P_{n}^{(1)}(\cos \theta)}{d \theta} \\
a_{n}=\frac{\Psi_{n}(x) \Psi_{n}^{\prime}(m x)-m \Psi_{n}^{\prime}(x) \Psi_{n}(m x)}{\xi(x) \Psi_{n}^{\prime}(m x)-m \xi_{n}^{\prime}(x) \Psi_{n}(m x)}
\end{gathered}
$$




$$
b_{n}=\frac{\mathbf{m} \Psi_{n}(x) \Psi_{n}^{\prime}(\mathbf{m x})-\Psi_{\mathbf{n}}^{\prime}(\mathbf{x}) \Psi_{\mathbf{n}}(\mathbf{m x})}{\xi(\mathbf{x}) \Psi_{\mathbf{n}}^{\prime}(\mathbf{m x})-\mathbf{m} \xi_{\mathbf{n}}^{\prime}(\mathbf{x}) \Psi_{\mathbf{n}}(\mathbf{m x})}
$$

Term $\mathrm{P}_{\mathrm{n}}^{(1)}$ is the Legendre polynomials, defined as

$$
\mathbf{P}_{n}(\mathbf{x})=2^{\mathbf{n}} \cdot \sum_{\mathrm{k}=\mathbf{0}}^{\mathbf{n}} \mathbf{x}^{\mathrm{k}}\left(\begin{array}{l}
\mathbf{n} \\
\mathrm{k}
\end{array}\right)\left(\frac{\mathbf{n}+\mathbf{k}-\mathbf{1}}{\mathbf{2}}\right)
$$

where both $\mathrm{n}$ and $\mathrm{k}$ are integers. $\mathrm{m}$ is the refractive index, $\mathrm{x}$ is dimensionless size parameter which is determined

$$
\mathbf{x}=\frac{\boldsymbol{\pi d}}{\boldsymbol{\lambda}}
$$

where $d$ is the diameter of scattered particle and $\lambda$ is the incident light wavelength. The Ricatti-Bessel function $\Psi$ and $\xi$ are defined as $[18,19,24,25]$

$$
\begin{aligned}
& \Psi_{n}(x)=\left(\frac{\pi x}{2}\right)^{\frac{1}{2}} J_{n+\frac{1}{2}}(x) \\
& \xi_{n}(x)=\left(\frac{\pi x}{2}\right)^{\frac{1}{2}} H_{n+\frac{1}{2}}^{(2)}(x)
\end{aligned}
$$

$\mathrm{J}_{\mathrm{n}+\frac{1}{2}}(\mathrm{x})$ is the half-integer order Bessel function in terms of the first kind. $\mathbf{H}_{\mathrm{n}+\frac{1}{2}}^{(2)}(\mathbf{x})$ is the half-integer order Henkel function of second kind.

Mie coefficient $\left(a_{n}\right.$ and $\left.b_{n}\right)$ can also expressed in the form as in equation (4.31) and (4.32) [17, 26]:

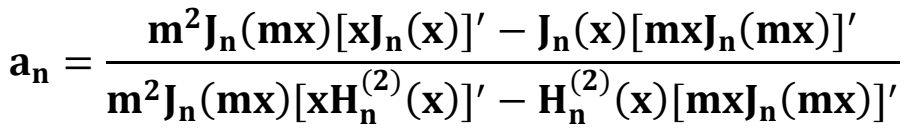

$$
\begin{aligned}
& \mathbf{b}_{\mathbf{n}}=\frac{\mathbf{J}_{\mathbf{n}}(\mathbf{m x})\left[\mathbf{x J}_{\mathbf{n}}(\mathbf{x})\right]^{\prime}-\mathbf{J}_{\mathbf{n}}(\mathbf{x})\left[\mathbf{m x} \mathbf{J}_{\mathbf{n}}(\mathbf{m x})\right]^{\prime}}{\mathbf{J}_{\mathbf{n}}(\mathbf{m x})\left[\mathbf{x H}_{\mathbf{n}}^{(2)}(\mathbf{x})\right]^{\prime}-\mathbf{H}_{\mathbf{n}}^{(2)}(\mathbf{x})\left[\mathbf{m x} \mathbf{J}_{\mathbf{n}}(\mathbf{m x})\right]^{\prime}}
\end{aligned}
$$

The light intensity of single particle scattering $I$ at unit solid angle $\theta$ with a distance $\mathrm{R}$ to the scattered particle is [19] [24] [25]

$$
\mathbf{I}=\frac{\lambda^{2} \mathbf{I}_{0}}{4 \pi^{2} \mathbf{R}^{2}}\left[\mathbf{i}_{1}(\theta)+\mathbf{i}_{2}(\theta)\right]
$$


where $I_{0}$ is the incident light intensity before scattering by the particle, $i_{1}$ and $i_{2}$ are the intensity functions have the relationship with scattering amplitude $S_{1}$ and $S_{2}$ as

$$
\mathbf{i}_{1}(\theta)=\left|\mathbf{S}_{1}(\theta)\right|^{2} \text { and } \mathbf{i}_{2}(\theta)=\left|\mathbf{S}_{\mathbf{2}}(\theta)\right|^{2}
$$

Equation (4.33) is used for single particle scattering. When the scattering happens at where multiple particle existing with the known number concentration $\mathrm{N}$, equation (4.33) is modified to

$$
I=\frac{N \lambda^{2} I_{0}}{4 \pi^{2} R^{2}}\left[i_{1}(\theta)+i_{2}(\theta)\right]
$$

Number concentration $\mathrm{N}$ represents the number of particles in a unit volume. In the calculation of Matlab program, $\mathrm{N}$ is considered as the number of particles in effective volume where light scatters in and scattered light by any particle is receivable to the photodiode. This effective volume is calculated in Solidworks by modeling a solid body for the desired volume, and then the volume of the body is shown through mass property as $0.15 \mathrm{~cm}^{3}$. With the effective volume known, the number concentration $\mathrm{N}$ is calculated by using the amount of particle in the effective volume divided by the effective volume.

Particle number concentration $\mathrm{N}$ is able to covert to particle mass concentration $\mathrm{W}$ with known particle density $\rho$ and diameter $\mathrm{d}$ :

$$
N=\frac{6 W}{\pi \rho d^{3}}
$$

\subsubsection{Resolution and Range of Detection Calculation by MatLab}

Mie theory is calculated with the help of MatLab. The program is modified based on the code originally developed by Christian Mätzler in the report "MatLab Functions for Mie Scattering and Absorption" [26]. The entire code are split into four files that named "Mie_concentration", "Mie-anbn", "Mie_pt" and "Mie_S1S2" responsible to calculating the mass concentration, Mie coefficient, Mie angular functions, and scattering amplitude, respectively. In the four files, "Mie concentration" is the directly calling function by a user, and the rest are functions which are called internally by the main function. 
The derived Mie Solution to Maxwell equations have infinite iteration of summations in the equation of scattering amplitude that is impossible to solve in mathematics. There is a suggestion on the amount of iteration $\mathrm{n}$ to Mie calculation based on the size parameter of the particle $\mathrm{x}$ (4.37) [27]:

$$
n=\left\{\begin{array}{c}
x+4 x^{\frac{1}{3}}+1 \quad 0.02 \leq x \leq 8 \\
x+4.05 x^{\frac{1}{3}}+2 \quad 8<x<4200 \\
x+4 x^{\frac{1}{3}}+2 \quad 4200 \leq x \leq 20000
\end{array}\right.
$$

Mie theory is suitable for computing scattering by homogenous spherical particles which have the same size and the same chemical composition. Density and refractive index are associated with chemical composition. In reality, aerosol is formed by different materials and/or combination of materials at wild range of size. The refractive index and average density of PM2.5 are 1.566 and $1.87 \mathrm{~g} / \mathrm{cm}^{3}$, respectively, and these values for PM2.5 and PM10 differ insignificantly [28]. Therefore, in the Mie calculation, the refractive index is fixed with the value of 1.566 and density used is $1.87 \mathrm{~g} / \mathrm{cm}^{3}$ (or $1870 \mathrm{mg} / \mathrm{m}^{3}$ ). For size of particle, the range was taken form $0.1 \mu \mathrm{m}$ to $10 \mu \mathrm{m}$ with increment of $0.1 \mu \mathrm{m}$. In regarding to the calculation of sensitivity and upper boundary of sensing range, the medium of the result was taken from the calculation within the size range selected.

Sensitivity of the design is calculated according to the real laser and photodiode parameter. The sensitivity of photodiode is $1.65 \mathrm{E}-10 \mathrm{~W}$. Having $1.65 \mathrm{e}-10 \mathrm{~W}$ as the value of received scattered light intensity, the resolution of the design is then calculated by applying Mie theory and result is $0.3238 \mu \mathrm{g} / \mathrm{m}^{3}$.

The range of mass concentration of the design is computed under the condition of two extreme cases: assuming no light scattered and received, and all light scattered and also received. The first assumption (no light scattered and received), is in equivalent to $I_{s}=0$, gives the lower boundary of the range which is $0 \mu \mathrm{g} / \mathrm{m}^{3}$. The second assumption (all light scattered and received), is in equivalent to $I_{s}=1 \mathrm{~mW}$, provides the upper boundary of the sensing range; however, which is $1.9624 \mathrm{e} 6 \mu \mathrm{g} / \mathrm{m}^{3}$ that will highly unlikely happen. Therefore, the range of the design with selected laser module and photodiode is from 0 to $1.9624 \mathrm{e} 6 \mu \mathrm{g} / \mathrm{m}^{3}$. 


\section{Chapter 5 Virtual Impactor}

In this Chapter, a virtual impactor will first be introduced. Aerodynamic size of particle is used to represent the diameter of a particle in this chapter. The dimension of each parameter used in the design is explained.

\subsection{Theory}

A virtual impactor is used to separate particles with a desired diameter from other sized particles. It takes advantage of different inertial forces for particles contained in the air flow for dividing particles into two separated channels. Large size particles cannot make sharp turns and therefore move straight into the collection probe (named minor flow), while small size particles with less inertia follow a curved air stream line and enter into side passage(s) (named major flow) [29, 30]. Figure 14 shows the schematic of a typical virtual impactor.

\subsection{Aerodynamic Diameter}

In reality, airborne particles cannot be in perfect spherical shape or other regular shape, which makes it hard to determine each particle based on its physical dimension. In order to separate particles based on their size, a common method in the field of aerosol science is used which is to find the aerodynamic equivalent dimension. The aerodynamic diameter of an irregular particle provides the diameter of the spherical particle with $1000 \mathrm{~kg} / \mathrm{m}^{3}$ density and the same settling velocity as the irregular particle [31]. The settling velocity (Vs) is expressed as:

$$
\text { Vs }=\tau \mathbf{g}
$$

where $\boldsymbol{\tau}$ is particle relaxation time and $\mathrm{g}$ is gravity. $\boldsymbol{\tau}$ of a given particle is calculated by the expression:

$$
\tau=\frac{D_{p}^{2} \rho_{p} C g}{18 \mu}
$$

where $D_{p}$ is particle diameter, $\rho_{p}$ is particle density, $C$ is slip correction associated with particle size, and $\mu$ is air viscosity. 
Since the settling velocity $(\mathrm{Vs})$ is the same to particle aerodynamic diameter $\left(\mathrm{D}_{\mathrm{pa}}\right)$ and the diameter of a sphere with density of $1000 \mathrm{~kg} / \mathrm{m}^{3}\left(\rho_{\mathrm{pa}}\right)$, the equation can be derived as [32]:

$$
D_{p a}=D_{p} \sqrt{\frac{\rho_{p} C}{\rho_{p a} C_{a}}}
$$

\subsection{Design of Virtual Impactor}

The procedure to determine the design dimension is [33]:

1. Determine a target cutoff size for particles, flow rate at nozzle throat, thickness of the channel, and the Stokes Number (Stk).

2. Calculate slip-correction factor.

3. Calculate nozzle width $\mathrm{W}$.

4. Verify Reynold Number (Re) to determine flow is within laminar regime $(\operatorname{Re}<1000)$.

The Stoke number ( $\mathrm{St}$ ) and Reynolds number $(\mathrm{Re})$ governs particle behavior and fluid behavior in the virtual impactor respectively [34]. Stoke's number is used to measure the dimensionless diameter. Reynolds number is the ratio of inertial force to viscous force of a fluid in a certain condition, which is important to indicate the flow is either laminar or turbulent.

Stoke's number (Stk) and Reynolds number (Re) are expressed as [33]

$$
\begin{aligned}
& \text { Stk }=\frac{\rho_{\mathrm{p}} \mathrm{VCD}_{\mathrm{p}}^{2}}{9 \mu \mathrm{W}} \\
& \mathrm{Re}=\frac{4 \rho \mathrm{WtU}}{\mu(\mathrm{W}+\mathrm{t})}
\end{aligned}
$$

where, $\rho_{\mathrm{p}}=$ the density of particle $\left(=1870 \mathrm{~kg} / \mathrm{m}^{3}\right)$,

$\mathrm{V}=$ mean air velocity at nozzle throat,

$\mathrm{C}=$ the Cunningham slip correction,

$\mathrm{D}_{\mathrm{p}}=$ the diameter of particle, 


$$
\begin{aligned}
& \mu=\text { the absolute air viscosity (Air }=18.6 \mathrm{e}-6 \mathrm{~kg} \mathrm{~m} / \mathrm{s} \text { at } 25^{\circ} \mathrm{C} \text { ), } \\
& \mathrm{w}=\text { nozzle width for rectangular jets, } \\
& \rho=\text { the density of fluid (Air }=1.1839 \mathrm{~kg} / \mathrm{m}^{3} \text { at } 25^{\circ} \mathrm{C} \text { ), } \\
& \mathrm{t}=\text { height of air channel. }
\end{aligned}
$$

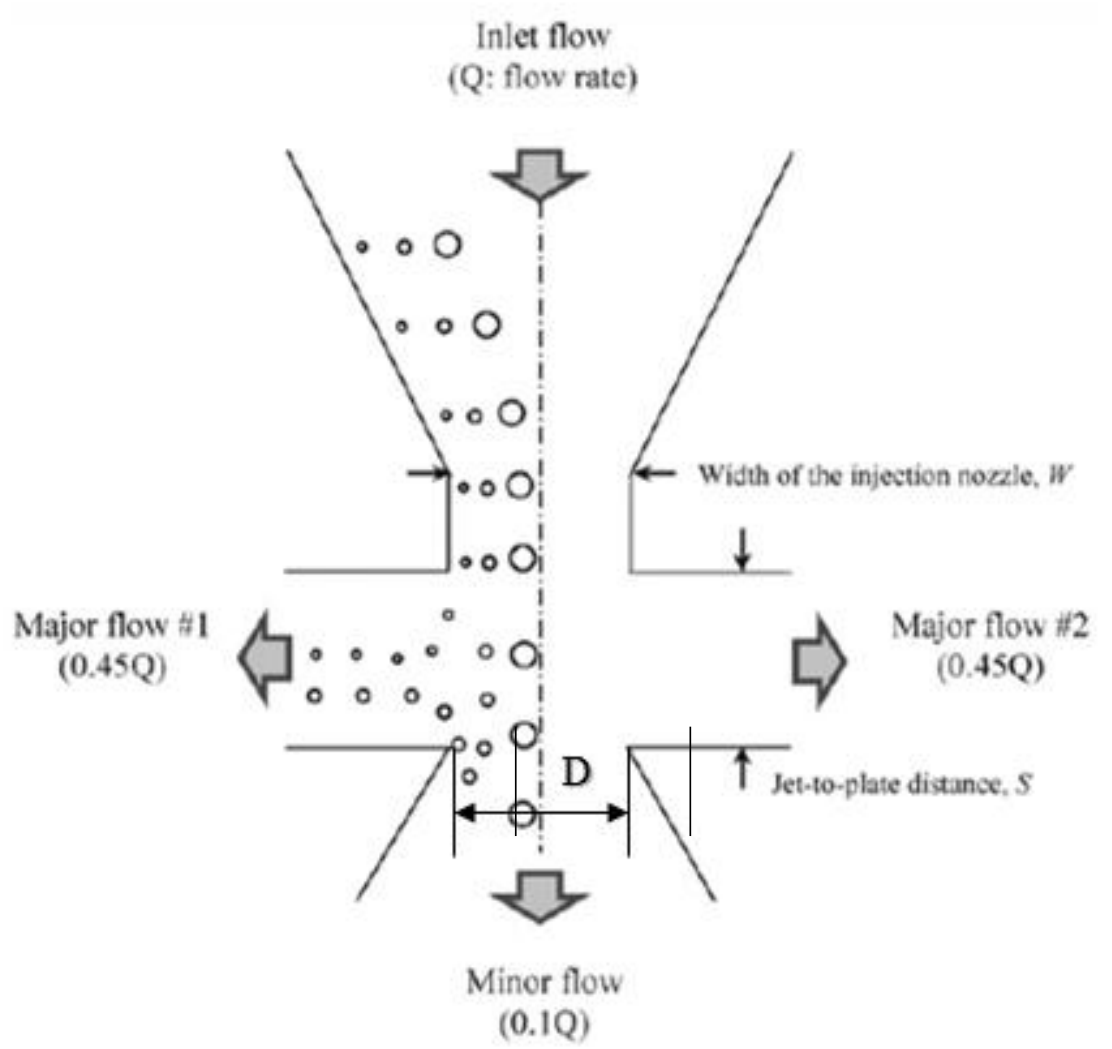

Figure 14 Virtual Impactor Cross-section View [33]

The cutoff size $\left(D_{p}\right)$ is 10 microns. The inlet flow rate $(q)$ and height of air channel $\mathrm{t}$ is set to $2 \mathrm{e}-5 \mathrm{~m}^{3} / \mathrm{s}$ and $5 \mathrm{~mm}$ respectively after serval attempts (adjusting $\mathrm{t}$ and $\mathrm{q}$ value to solve $\mathrm{w}$ and $\mathrm{v}$ for laminar flow). Stk50 of 0.709 is adopted for its low particle loss by experimental for $10 \mu \mathrm{m}$ cutoff size [32]. Stk50 is the Stk at $50 \%$ collection efficiency of a particle, which corresponds to the cutoff size. Slip correction number (C) is 1.0166 determined by equation:

$$
C=1+1.257 \frac{2 \lambda}{D_{p}}+0.4 \frac{2 \lambda}{D_{p}} \exp \left(-1.1 \frac{D_{p}}{2 \lambda}\right)
$$

where $\lambda$ is mean free path of an air molecule $(=6.6 \mathrm{e}-8 \mathrm{~m})$ [33]. Deriving equation (5.4) can get the equation for nozzle width parameter $(\mathrm{W})$ as 


$$
W=\sqrt{\frac{\rho_{\mathrm{p}} \mathrm{D}_{\mathrm{p}}^{2} \mathrm{qC}}{9 \mu \mathrm{Stk}_{50} \mathrm{t}}}
$$

where $\mathrm{q}$ is volumetric flow rate at nozzle $\left(3 \mathrm{e}-5 \mathrm{~m}^{3} / \mathrm{s}\right.$ ) (equation (5.8)) and therefore $\mathrm{W}$ is $0.0031 \mathrm{~m}(3.1 \mathrm{~mm})$.

$$
\mathbf{V}=\frac{\mathbf{q}}{\mathbf{w t}}
$$

Reynold number is 942.9635 with calculated parameter above and the flow is remains in the laminar regime. The ratio of $\mathrm{S}$ (jet-to-plate distance) to $\mathrm{W}$ is better within the range of 1.2 to 1.8 [33]. Therefore, $\mathrm{S}$ is $4.5 \mathrm{~mm}$ when ratio of 1.5 is set. Collection probe entrance width, D (Figure 14), is suggested to be larger than $\mathrm{W}$ by a factor of around 1.33 and less than 1.44 for less particle loss [30]. Thus, D is $4.34 \mathrm{~mm}$ with chosen factor of 1.4 .

\subsection{Particle Loss}

Ideally, particles carried by air flow entering into a virtual impactor exit either at a side passage (major flow) or a collection probe (minor flow). In fact, based on experimental data, there is less amount of particles exited comparing to the amount of particles entered. After study, it is believed that there is particle loss in the impactor, which is those particles that impact on impactor inner walls. Particle loss will result in inaccurate measurements unless it is compensated or minimized to the level which can be mathematically negligible. The conception of particle loss is defined as when a particle comes to a wall that the distance between the wall and the center of the particle is less than the radius of the particle. There are two methods to determine a particles trajectory (exit at collection probe, impact on inner wall, or exit at side passage). One is having $\sqrt{\mathrm{St}}$ unchanged but let particles entering at different streamlines. In this method, one can find the threshold to particle loss in terms of streamline (for example, particle loss happens at between $20 \%$ and $70 \%$ streamline for particle has size of $\sqrt{\mathrm{St}}=0.5$ [30]. Another method is to let all particles entering at a fixed streamline but change $\sqrt{\mathrm{St}}$ every experiment. A $\sqrt{\mathrm{St}}$ range will be found, which indicates the particle size range will cause particle loss at a certain streamline (for example, particle with size $\sqrt{\text { St }}$ greater than 0.677 
but less than 0.881 will impact on collection probe wall at 50\% streamline [30]. Figure 15 shows the grid and streamlines used to determine collection efficiency of base condition $\left(\mathrm{Re}=5000, \mathrm{Q}_{1} / \mathrm{Q}_{0}=0.10, \mathrm{D}_{1} / \mathrm{D}_{0}=1.33, \mathrm{~L}_{0} / \mathrm{D}_{0}=2.5, \theta_{0}=45^{\circ}\right.$, and collection probe is thin wall type (as seen in Figure 15 (b)).

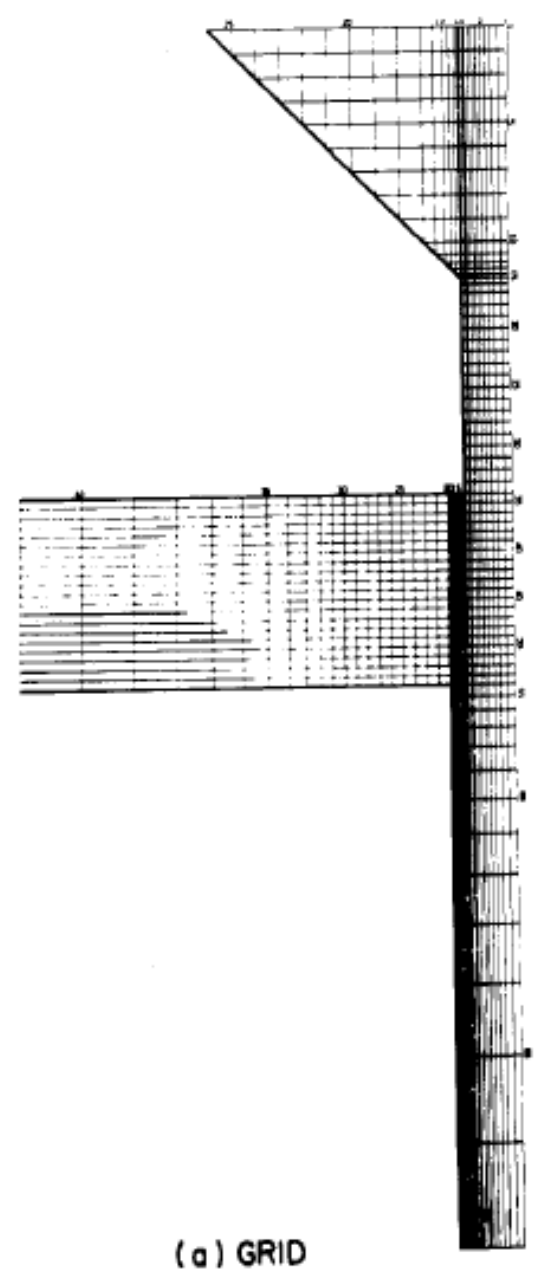

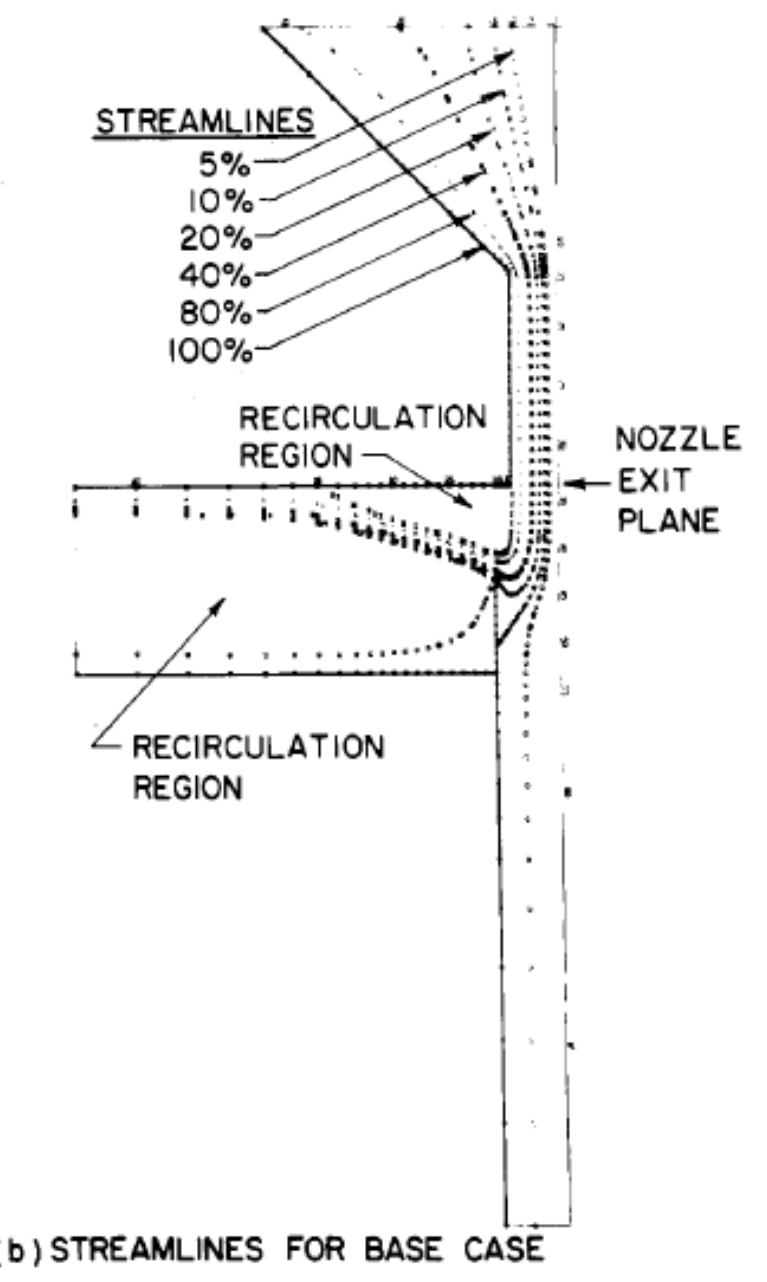

(b) STREAMLINES FOR BASE CASE

Figure 15 (a) Grid used to determine particle trajectory in virtual impactor and (b) Particle trajectory when entering by different streamline at the base condition [30] 


\section{Chapter 6 Prototyping and Testing}

In this Chapter, the method of prototyping assembly will be presented. A testing rig is designed to conduct experiments for testing the developed unit's performance. Setup of experiment and procedure of operation will be discussed in section two.

\subsection{Prototyping}

There will be four essential components for the prototype: a fan, a laser module, a photodiode, and a fluid channel containing a cover (Figure 16) and a base (Figure 17 and Figure 18). The fluid channel will be made using latest 3D printing technology. On both parts of the fluid channel (cover and base), cuts for optical units (Laser and photodiode) were according to their real dimensions and therefore the structure of the fluid channel limits the movements of the light emitter and detector. Screw holes were included for fluid channel fastening and fan mounting.

Figure 16 shows the cover of the virtual impactor (air channel). Figure 17 and Figure 18 show the base of the virtual impactor and inner structure is showed in section view, respectively. Figure 19 gives the assembly of all part, and Figure 20 demonstrate how laser module and photodiode are assembly with the air channel.

Table 2 shows the dimension and power of each component selected to current design.

Table 2 Dimension and Power of Each Component

\begin{tabular}{|l|l|l|}
\hline & Dimension & Power \\
\hline Fan & $15 \mathrm{~mm}^{*} 15 \mathrm{~mm} * 4.5 \mathrm{~mm}$ & $0.3 \mathrm{~W}$ \\
\hline Laser Module & $8 \mathrm{~mm}(\mathrm{dia}) * 36 \mathrm{~mm}$ & $0.15 \mathrm{~W}($ Max $)$ \\
\hline Photodiode & $5.4 \mathrm{~mm}(\mathrm{dia}) * 3.6 \mathrm{~mm}$ & $0.05 \mathrm{~W}($ Max $)$ \\
\hline Air Channel & $56 \mathrm{~mm}(\mathrm{~L}) * 36 \mathrm{~mm}(\mathrm{~W}) * 12 \mathrm{~mm}(\mathrm{H})$ & \\
\hline
\end{tabular}




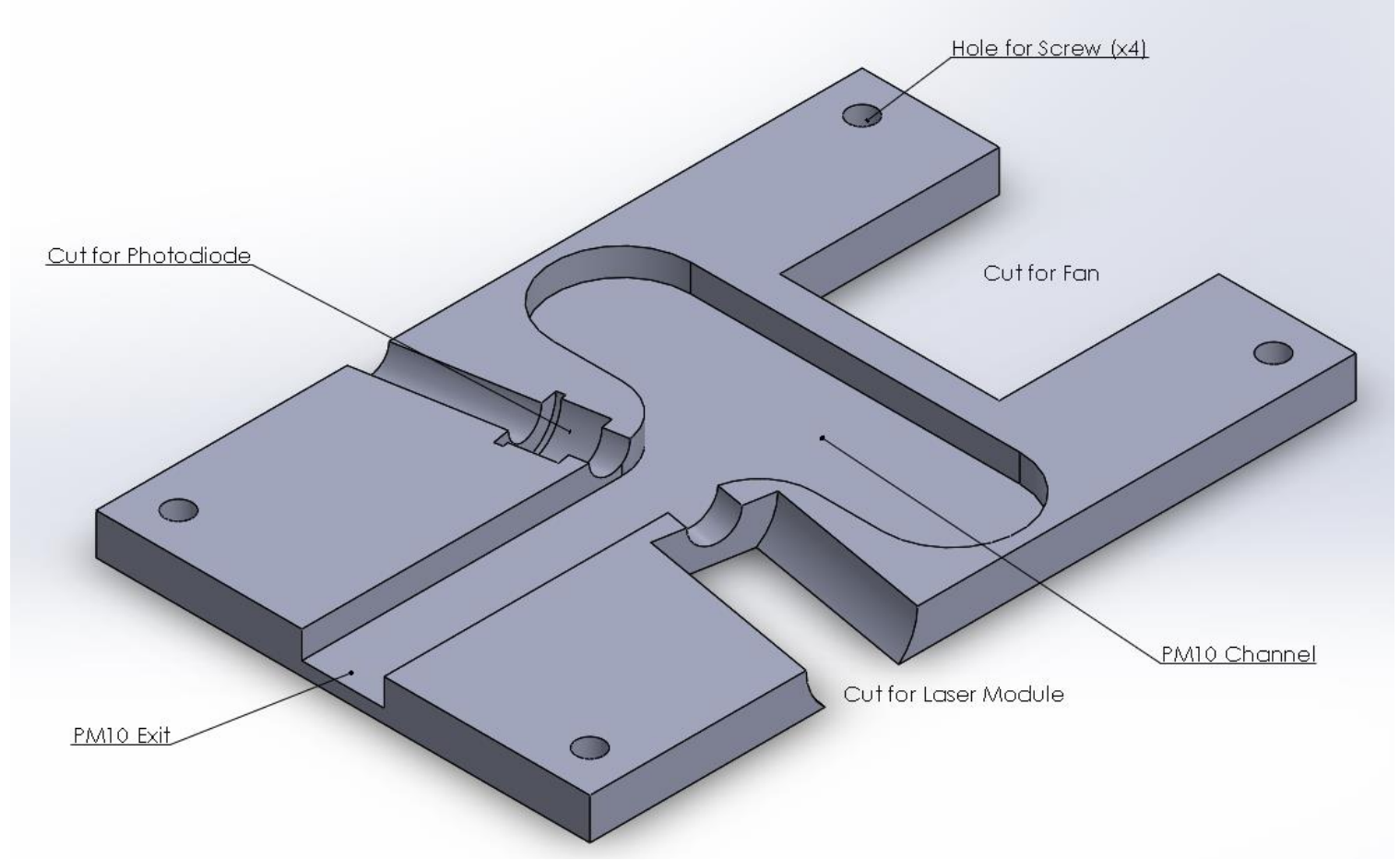

Figure 16 Virtual Impactor Base

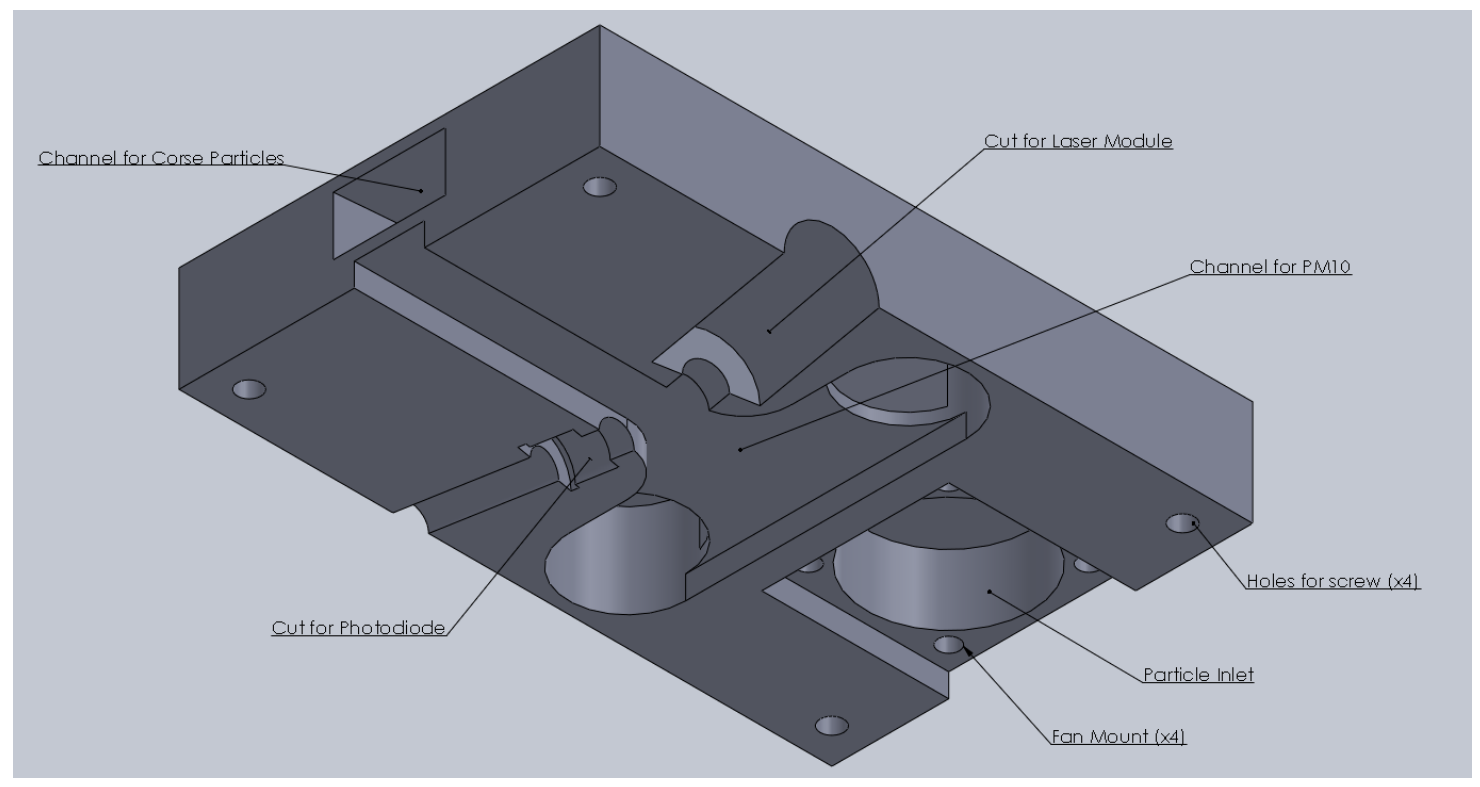

Figure 17 Virtual Impactor Cover (Flipped) 


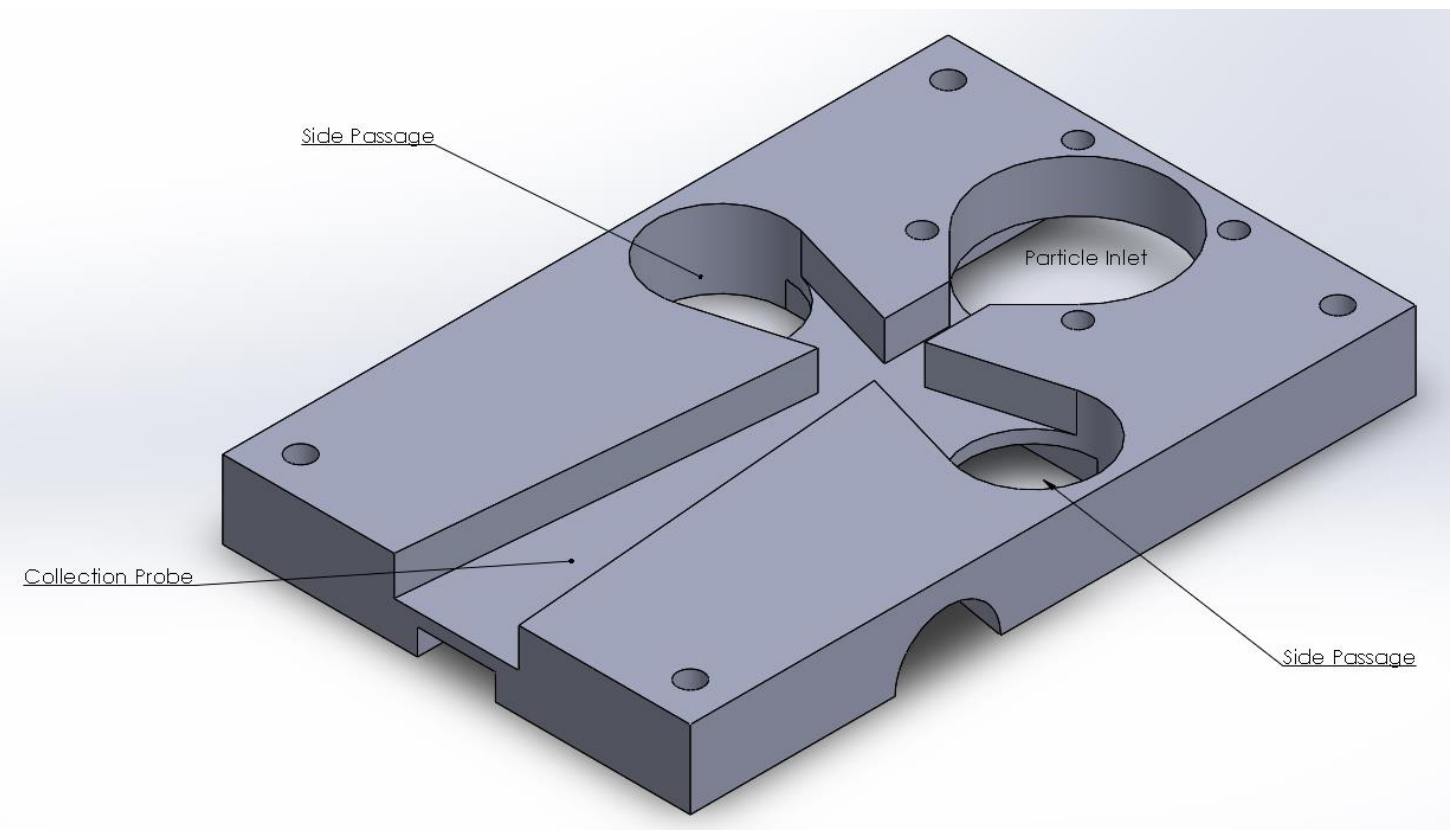

Figure 18 Virtual Impactor Cover (Section View)

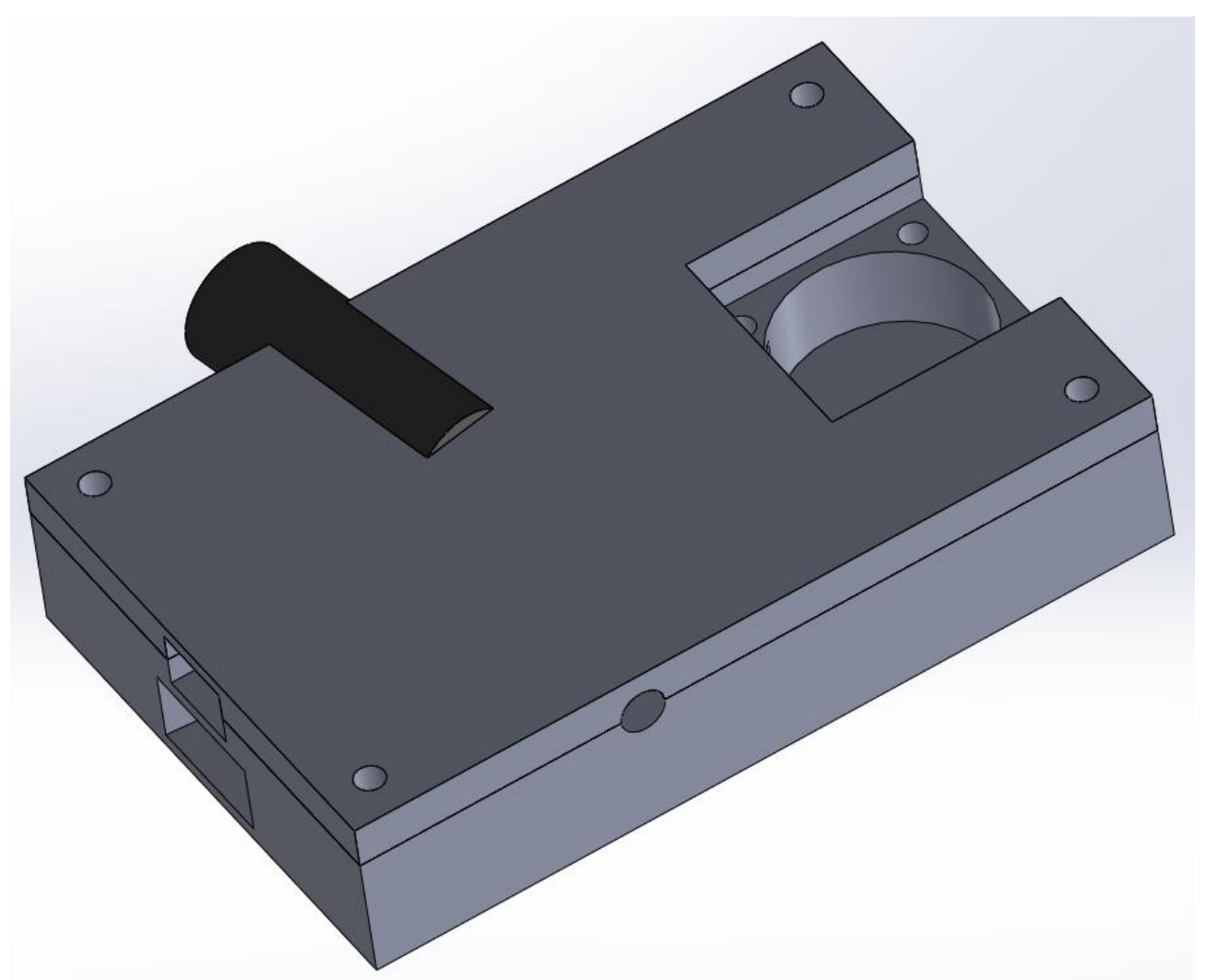

Figure 19 Assembly of Virtual Impactor 


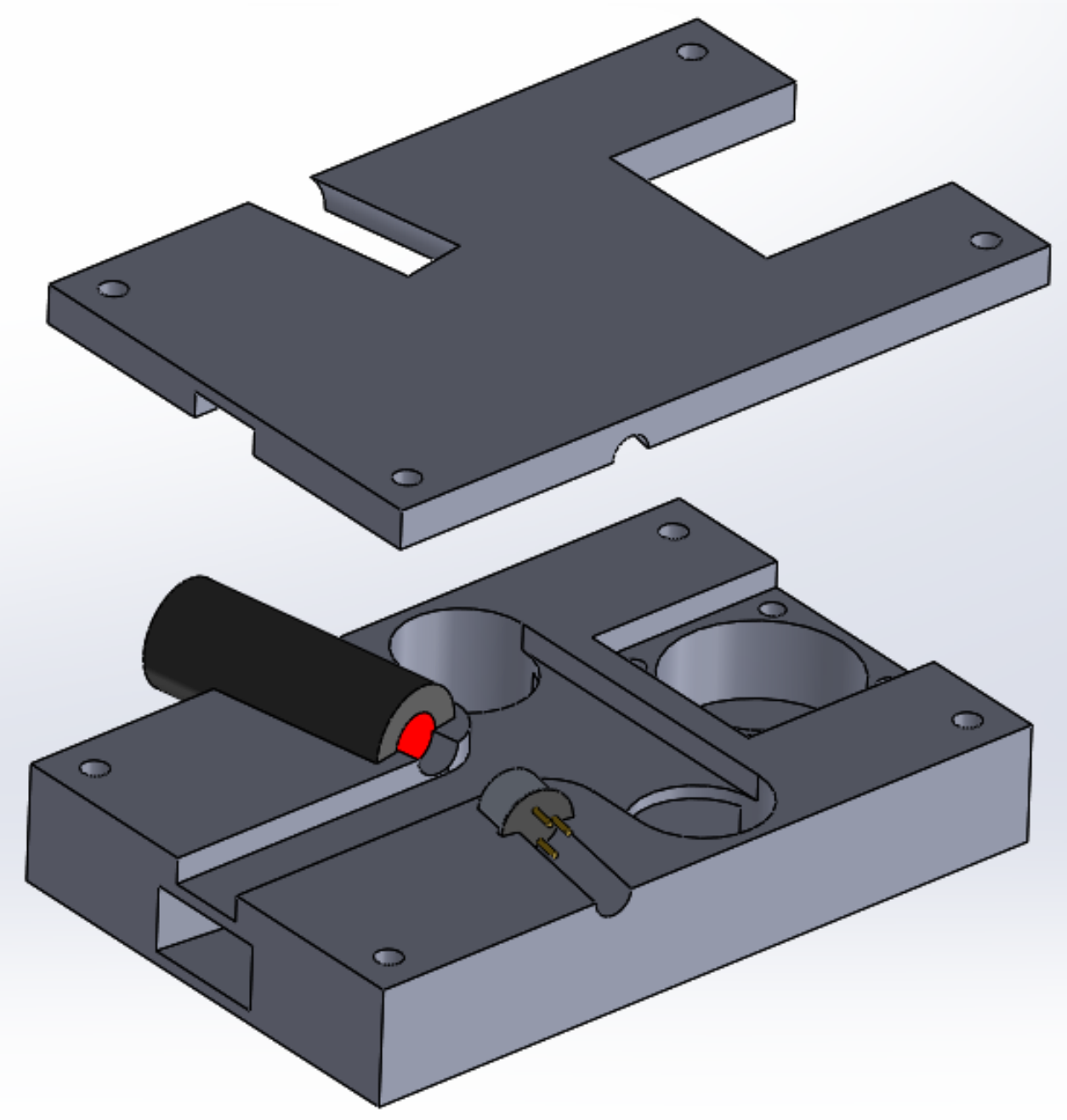

Figure 20 Explode View of Assembly of Virtual Impactor (Base and Cover) along with Laser Diode (Collimated) and Photodiode

\subsection{Testing}

In order to testify the performance of the design, a commercial PM10 monitor will be used as a comparison monitor. The difference between the two detected results will reveal if the measurements by designed PM10 detector are effective. Power of all electric components (fan, laser module, and photodiode) will be supplied by a laboratory DC power supply, and the signal from photodiode will be displayed on an oscillate scope after being amplified. 
Two groups of test will be conducted. In one group, same sized particles will enter the system in each experiment. In the other group of experiments, multiple sized particle mixed with various combination will be used in each experiment.

In order to simulate aerosols, some of the materials are selected to represent aerosol with different chemical composition and physical size such as cigarette smoke, baby powder, and sand dust. Considering the fact that fog scatters light, the experiment would give more effective results in a dry environment. Moreover, a clean room can eliminate the inaccuracy caused by unknown particle in the surrounding.

\subsubsection{Uniform Sized Particle Experiments}

In this group of experiments, one sized particle will be used in each experiment and data of the voltage on the oscillate scope will be recorded. The sensitivity to different sized particle of the scattering unit can be studied, and the minimum detection concentration to a given particle size will be figured. Collection efficiency of the virtual impactor will provide a parameter for error analysis.

\subsubsection{Multiple Sized Particle Experiments}

Different sized particle will be used, and data is recorded in each experiment. This group of experiments will mainly contribute on the study of the separation ability of virtual impactor. Light scattering behavior on multiple sized particle scattering will be analyzed to enhance designed PM10 detector.

\subsubsection{Idea of Testing Apparatus and Setup}

A testing rig is modeled for minimizing unknown environment influence. The rig has one end opened to connect an air purifier using in self-cleaning process if using in an indoor condition. The lid is removable by sliding, and therefore dust or other possible coarse particles may be added for testing at any time. Transparent material makes operator easy to control situation of test. The commercial PM monitor lays aside of the designed PM detector on the bottom of the testing rig for the most valid comparison result. The cut on the bottom of the rig is designed for protecting damage from any material that has to be heated or burned. Laying the rig on a flat surface with the lid will 
provide a sealed environment for testing. Figure 21 shows a simple model of testing rig designed for achieve all concerns mentioned above.

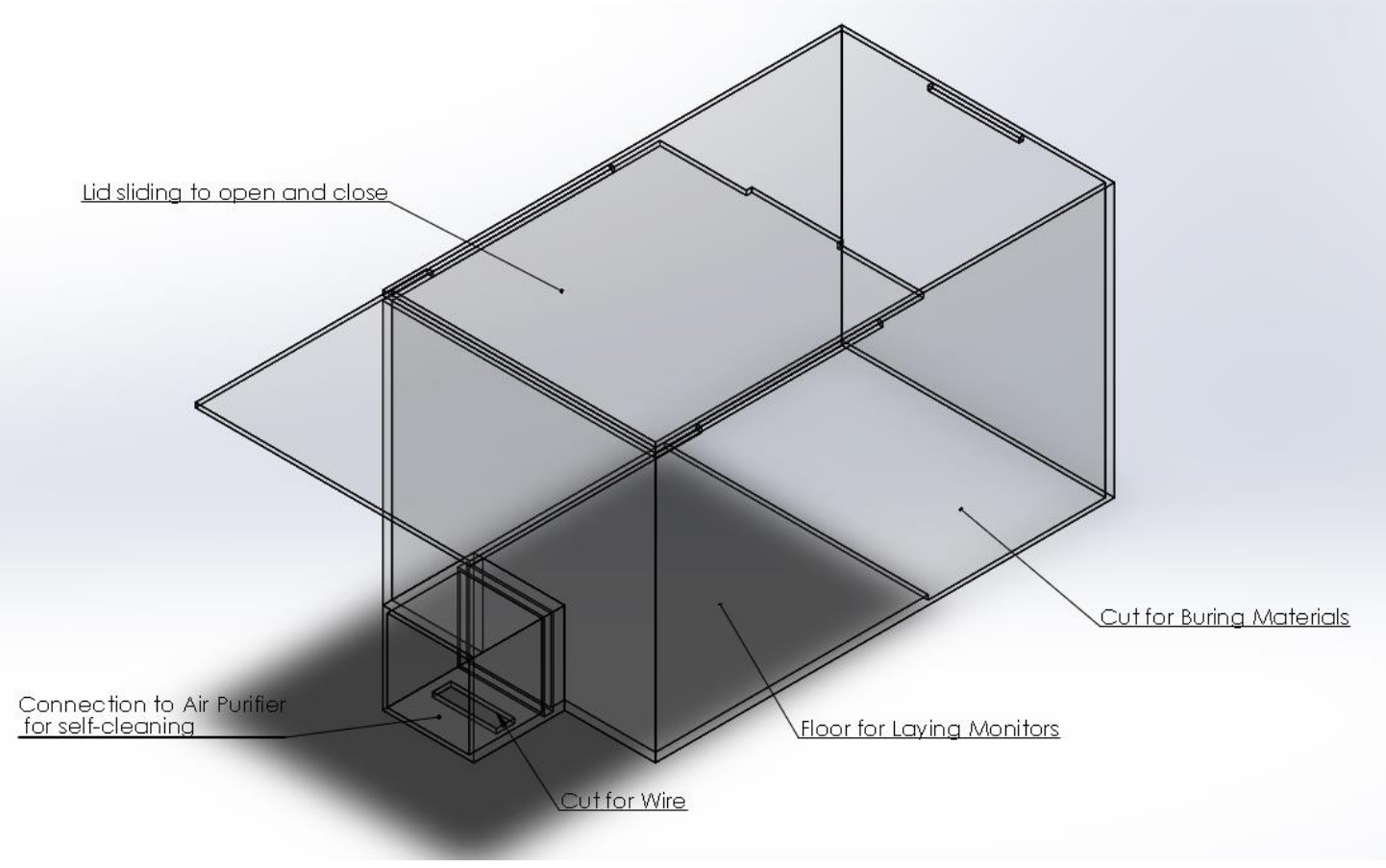

Figure 21 Testing Rig Model 


\section{Chapter 7 Conclusion}

\subsection{Contribution}

- A miniature PM10 detection unit is developed

The entire design contains four components: an air channel, a laser emitter, a photodiode and a fan. The special designed air channel plays two roles: one is being a virtual impactor that separates PM10 from other sized aerosols; and the other is itself is a plate where other components will mount at. The overall dimension achieved one-fifth of the size of the smallest commercial product in the market. The size is possible to be further reduced by changing to smaller fan and replacing laser module by laser diode.

- Low power consuming fan was selected, which dominates most of power of all components. Instantaneous measurement result is provided upon a display module (screen of a cell phone).

- Inexpensive components were chosen while accuracy and sensitivity are ensured. A ten level air quality indicator is achievable, and the monitoring unit covers a good sensing range. A few components involved would reduce the cost of manufacturing and assembly process.

- $\quad$ Procedure of test were presented and discussed.

\subsection{Future Work}

Experiments will be conducted and calibration will be done based on experimental result. Corresponding circuitry to drive laser diode and photodiode signal amplifier will preferably be designed or customized for better noise cancellation in order to record with higher resolution. The method of generating air flow may be replaced by a piezoelectric fan instead of a regular rotary fan for both size and energy optimization. The humidity and temperature influence on the error of light scattering phenomenon will be studied and generalized in order to expend operation condition of the design. A phone application may be developed for displaying measurement result and data can be recorded for future reference. 


\section{References}

[1] J. Chai, Director, Under the Dome. [Film]. China.2015.

[2] P. Baron, "Generation and Behavior of Airborne Particles (Aerosols)," National Institute for Occupational Safety and Health Centers for Disease Control and Prevention,Division of Applied Technology.

[3] S. Kong, B. Lu, Y. Ji, X. Zhao, Y. Xu, Y. Liu and H. Jiang, "Risk assessment of heavy metals in road and soil dusts within PM2.5, PM10 and PM100 fractions in Dongying city, Shandong Province, China," Journal of Environmental Monitoring, vol. 14, no. 3, pp. 791-803, 2012.

[4] "Particulate Matter (PM)," United States Environmental Protection Agency, 183 2013. [Online]. Available: http://www.epa.gov/oar/particlepollution/. [Accessed 23 June 2015].

[5] Eastern Technical Associates, "Visible Emissions Field Manual EPA Method 9 and 22," EPA, Raleigh, NC.

[6] D. Soldo, A. Quarto and V. Di Lecce, "M-DUST: An innovative low-cost smart PM sensor," in Instrumentation and Measurement Technology Conference (I2MTC), 2012 IEEE International, vol., no., pp.1823,182, Graz, 2012.

[7] K. B. Roberge, "Gravity and Cyclonic Separation of Bidispersed Suspensions," Department o f Chemical and Materials Engineering, University of Alberta, Edmonton, Alberta, Fall 2005.

[8] I. Paprotny, R. M. White and F. Doering, "MEMS Particulate Matter (PM) monitor for cellular deployment," in Sensors, 2010 IEEE, Kona, HI, 2010.

[9] J. P. Black, A. Elium, R. M. White, M. G. Apte, L. A. Gundel and R. Cambie, "MEMS-Enabled Miniaturized Particulate Matter Monitor Employing $1.6 \mathrm{GHz}$ Aluminum Nitride Thin-Film Bulk Acoustic Wave Resonator (FBAR) and Thermophoretic Precipitator," in Ultrasonics Symposium, 2007. IEEE, New York, NY, 28-31 Oct. 2007.

[10] Z. Cui, Y. Cui and X. Zhu, "Measuring equipment of dust mass concentration based on infrared absorption method," Journal of Henan Institute of Science and Technology, vol. 41, no. 2, pp. 70-74, Apr. 2013.

[11] E. Zhao, J. Sui, Z. Wang and Z. Li, "Charge Measurement of Dust Concentration Technology," Metal Mine, vol. Suppl., pp. 757-760, Novermber 2009. 
[12] L. Zhao, Y. Zhang, L. L. Christianson and G. L. Riskowski, "Measurement of Aerosol Concentration and Transportation in Ventilated Airspaces Using Particle Image Techniques," in ASAE, Orlando, Florida, 1998.

[13] D. S. Ensor, "Aerosol Science and Technology: History and Reviews," in History of Virtual Impactors, NC, RTI INternational, 2011, pp. 509-528.

[14] V. Mackowiak, J. Peupelmann, Y. Ma and A. Gorges, "Photodiode Tutorial," 2015. [Online].

Available: https://www.thorlabs.com/images/TabImages/Noise_Equivalent_Power_White_Pap er.pdf.

[15] Hamamatsu, "Si photodiode," Hamamatsu, 2015. [Online]. Available: http://www.hamamatsu.com/jp/en/product/category/3100/4001/4103/S122618BK/index.html. [Accessed 22 August 2015].

[16] D. W. Hahn, "Light Scattering Theory," Department of Mechanical and Aerospace Engineering, University of Florida, Florida, July 2009.

[17] L. N. Ng, "Manipulation of particles on optical waveguides," UMI Dissertations Publishing, Southampton UK, 2000.

[18] D. W. Hahn, "Rayleigh/Mie Light Scattering," July 2009. [Online]. Available: http://plaza.ufl.edu/dwhahn/Rayleigh\%20and\%20Mie\%20Light\%20Scattering.pdf.

[19] C. Hu, "Investigation of Dust Measuremnet Based on Scattering Theory," Soochow University, Suzhou, Jiangsu, 2007.

[20] I. N. Sokolik, "Atmospheric Radiative Transfer," 8 October 2009. [Online]. Available: http://irina.eas.gatech.edu/EAS8803_Fall2009/Lec14.pdf. [Accessed 16 August 2015].

[21] G. D. Fowler, "Measuring Suspended Sediment Characteristics to Identify Accurate Monitoring Techniques in Stormwater Runoff," University of New Hampshire, Durham, NH, 1999.

[22] I. N. Sokolik, "Remote sensing of the atmosphere and oceans," 7 Feb 2011. [Online]. Available: http://irina.eas.gatech.edu/EAS6145_Spring2011/Lecture4.pdf.

[23] I. N. Sokolik, "Atmospheric Radiative Transfer," 13 October 2009. [Online]. Available: http://irina.eas.gatech.edu/EAS8803_Fal12009/Lec15.pdf.

[24] P. Bradshaw, "Light scattering functions for particles used in the determination of smoke plume concentration," Journal of Aerosol Science, vol. 6, no. 2, pp. 147 156, 1975. 
[25] J. Liu and J. Wu, "Analysis of angular spectrum of scattering intensity with particles for laser beam," Optical Technique, vol. 31, no. 5, pp. 659-665, 2005.

[26] C. Mätzler, "MATLAB Functions for Mie Scattering and Absorption," Institute of Applied Physics, University of Bern, Bern, Switzerland, 2002.

[27] W. J. Wiscombe, "Improved Mie scattering algorithms," Applied Optics, vol. 19, no. 9, pp. 1505-1509, 1980.

[28] J. L. Hand and S. M. Kreidenweis, "A New Method for Retrieving Particle Refractive Index and Effective Density from Aerosol Size Distribution Data," Aerosol Science and Technology, vol. 36, no. 10, pp. 1012-1026, 2010.

[29] D. Liang, W.-P. Shih, C.-S. Chen and C.-A. Dai, "A Miniature System for Separating Aerosol Particles and Measuring Mass Concentrations," Sensors, vol. 10, no. 4, pp. 3641-3654, 2010.

[30] V. A. Marple and C. M. Chien, "Virtual Impactors: A Theoretical Study," Environmental Science \& Technology, vol. 14, no. 8, pp. 976-985, 1980.

[31] J.-H. Park, "Impactors and Particle Size Distribution," [Online]. Available: http://www.industrialventilation.net/ClassDownloads/IHS_725/L11\%20Impactor\%2 0Data\%20Reduction/Impactor_Park\%20(1).pdf. [Accessed 8 July 2015].

[32] Z. Xu, "A Study of Virtual Impactor," University of Minnesota, Minneapolis, MN, 1991.

[33] Y.-H. Kim, D. Park, H. Hwang and Y.-J. Kim, "A hybrid chip based on aerodynamics and electrostatics for the size-dependent classification of ultrafine and nano particles," Lab on a Chip, pp. 2722-2728, 2009.

[34] J. S. Haglund, "TWO LINEAR SLOT NOZZLE VIRTUAL IMPACTORS FOR CONCENTRATION OF BIOAEROSOLS," Texas A\&M University, College Station, TX, 2003.

[35] SEPA-Europe, "MF_15B05," 2011. [Online]. Available: http://www.sepaeurope.com/en/fan/axial/mf_15b05.

[36] Hamamatsu, "Si photodiode," 2015. [Online]. Available: http://www.hamamatsu.com/resources/pdf/ssd/si_pd_circuit_e.pdf. [Accessed 22 August 2015].

[37] World Star Tech, "UL-SERIES INFRARED LASER," January 2015. [Online]. Available: http://worldstartech.com/uploads/series/20140205212617_image_ML51G-650.pdf. 
[38] A. Morpurgo, F. Pedersini and A. Reina, "A low-cost instrument for environmental particulate analysis based on optical scattering," in Instrumentation and Measurement Technology Conference (I2MTC), 2012 IEEE International, Graz, 1316 May 2012.

[39] Environmental Protection Agency of United States , "National Air Quality and Emissions Trends Report, 1999," 1999. [Online]. Available: http://www.epa.gov/air/oaqps/airtrends/aqtrnd99/. [Accessed 21 July 2015]. 


\section{Appendix I - MatLab Program}

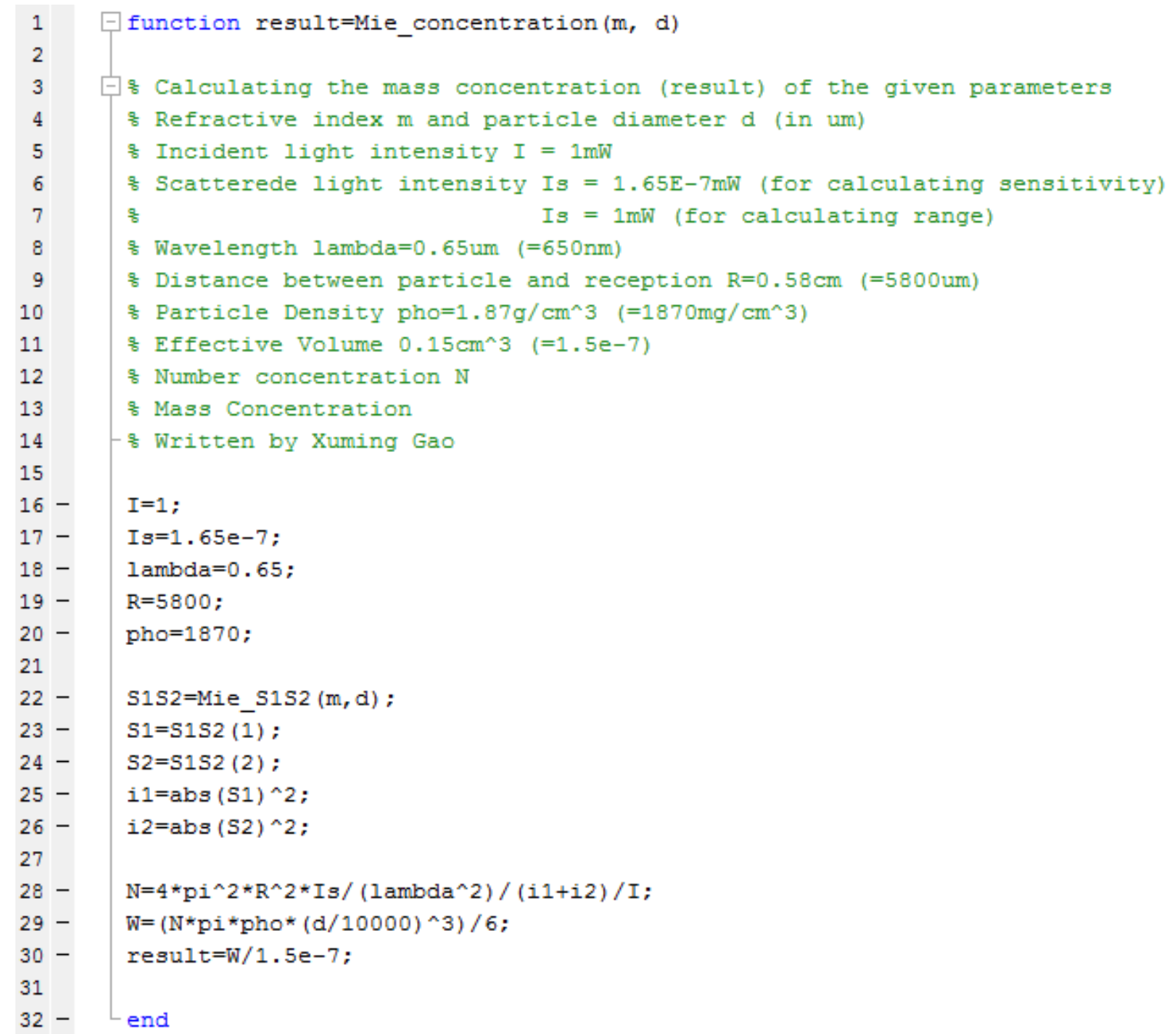

Figure 22 MatLab Program for Sensitivity Calculation

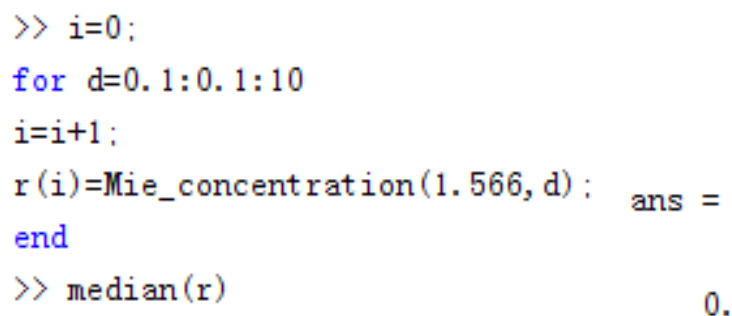

Figure 23 Command for Calculating the Median of Sensitivity and its Result 


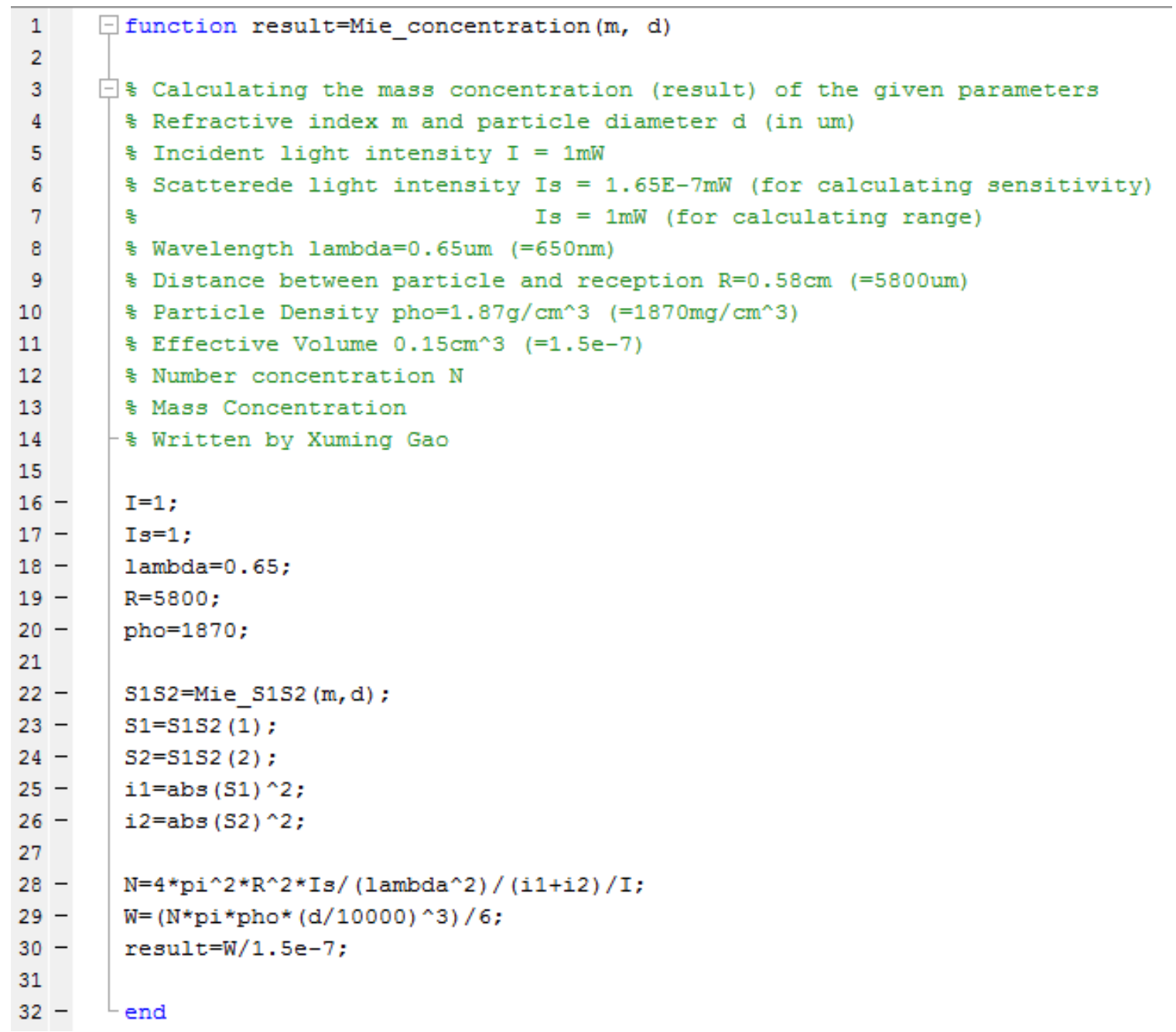

Figure 24 MatLab Program for Calculation of Upper Boundary of Sensing Range

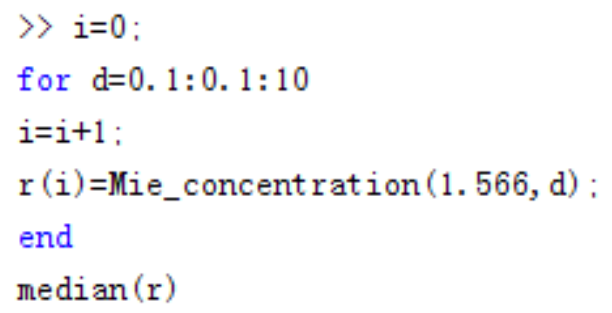

Figure 25 Command for Calculating the Median of Upper Boundary of Sensing Range and its Result 


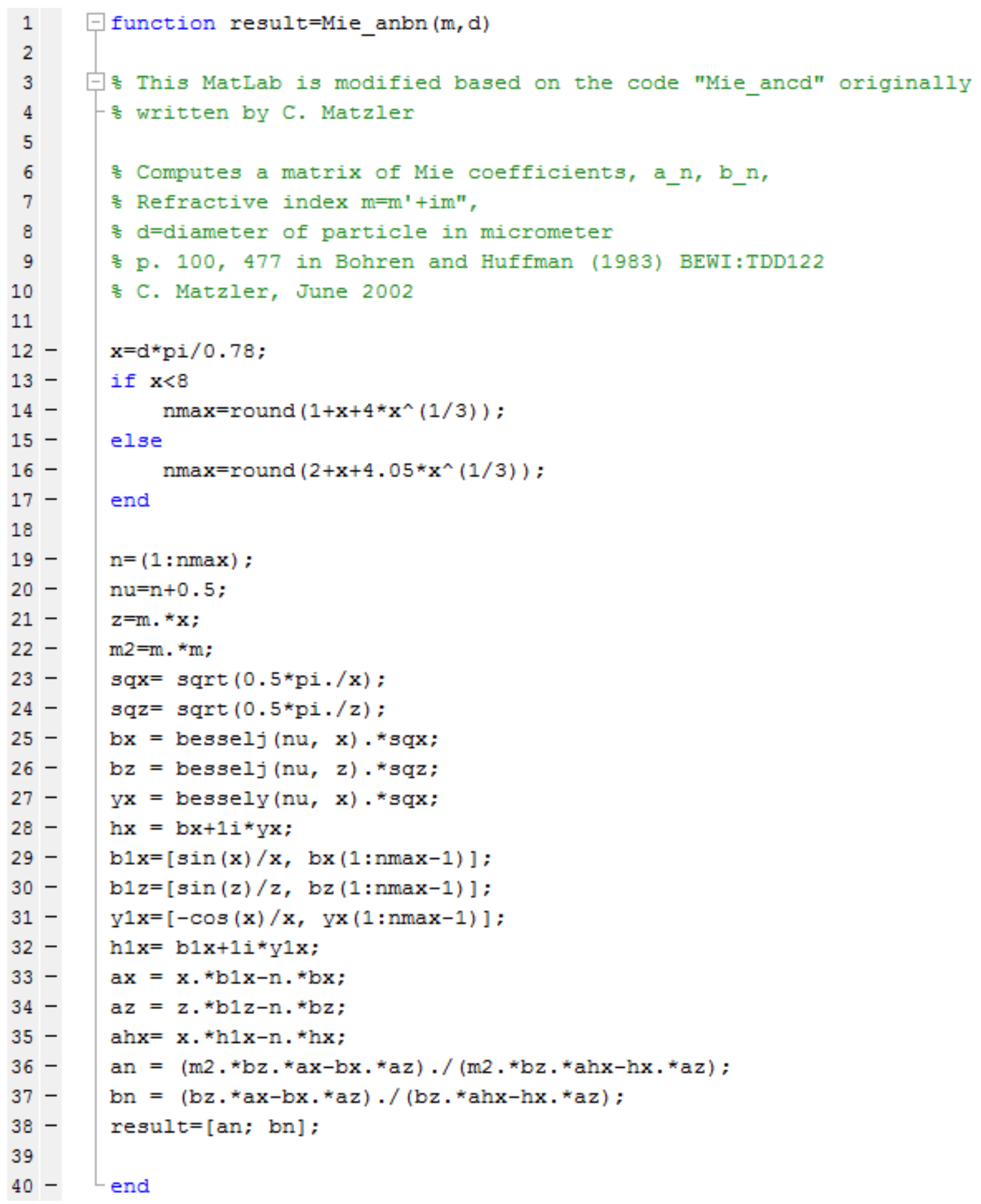

Figure 26 MatLab Program for Mie Coefficient Calculation [26] 


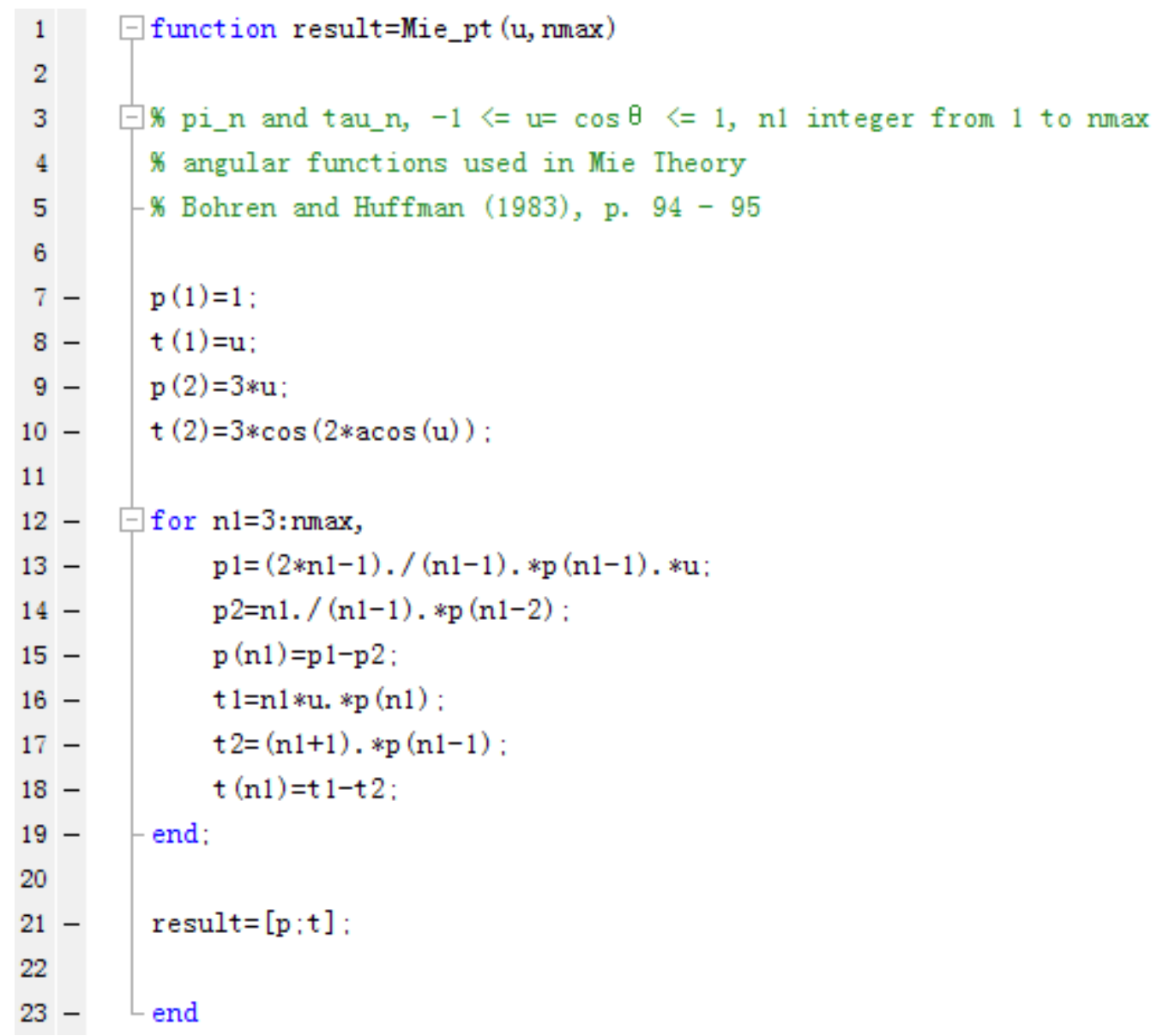

Figure 27 MatLab Program for Mie Angular Functions [26] 


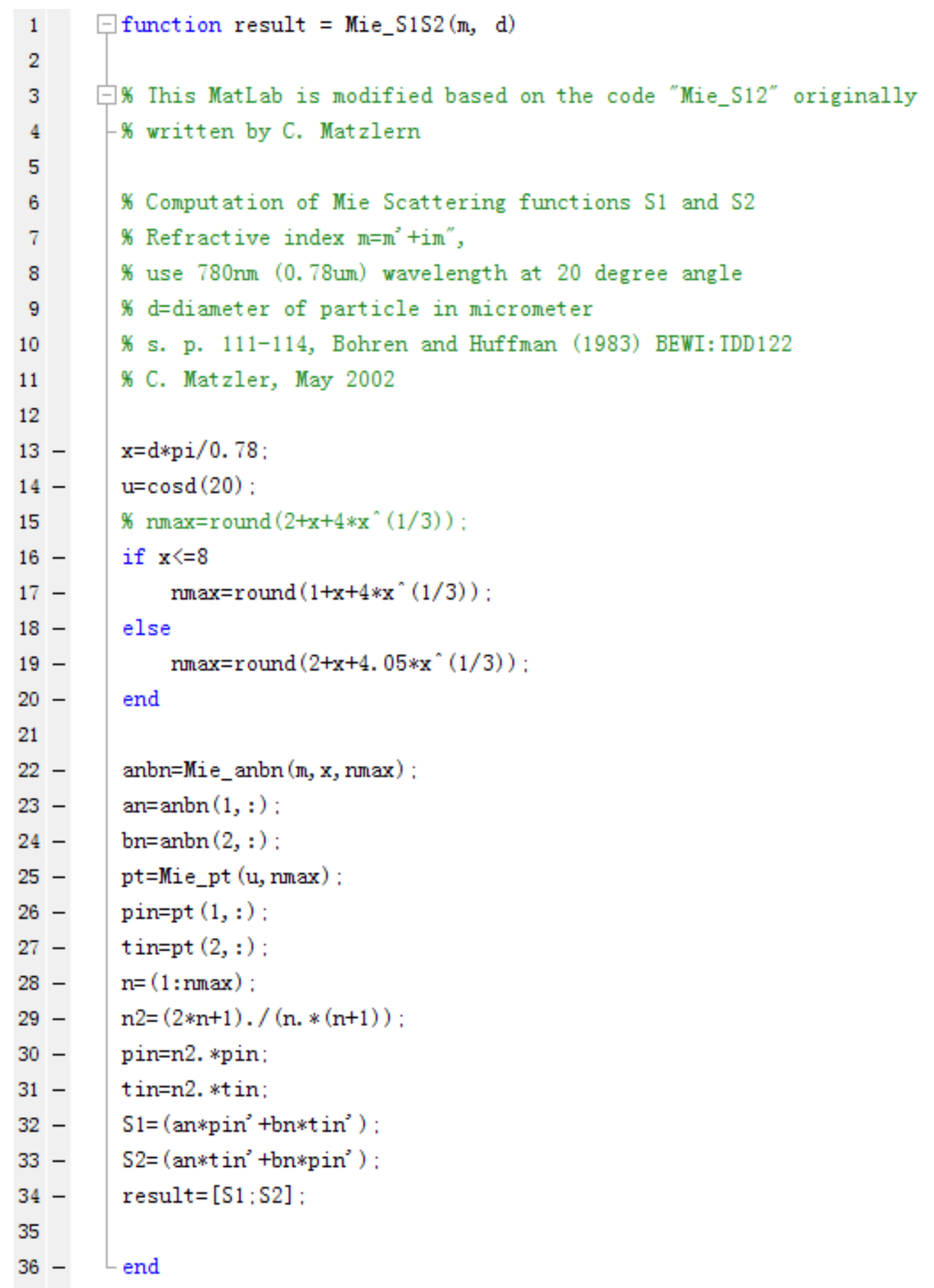

Figure 28 MatLab Program for Scatter Amplitude Calculation [26] 


\section{Appendix II - Selected Component Specification}
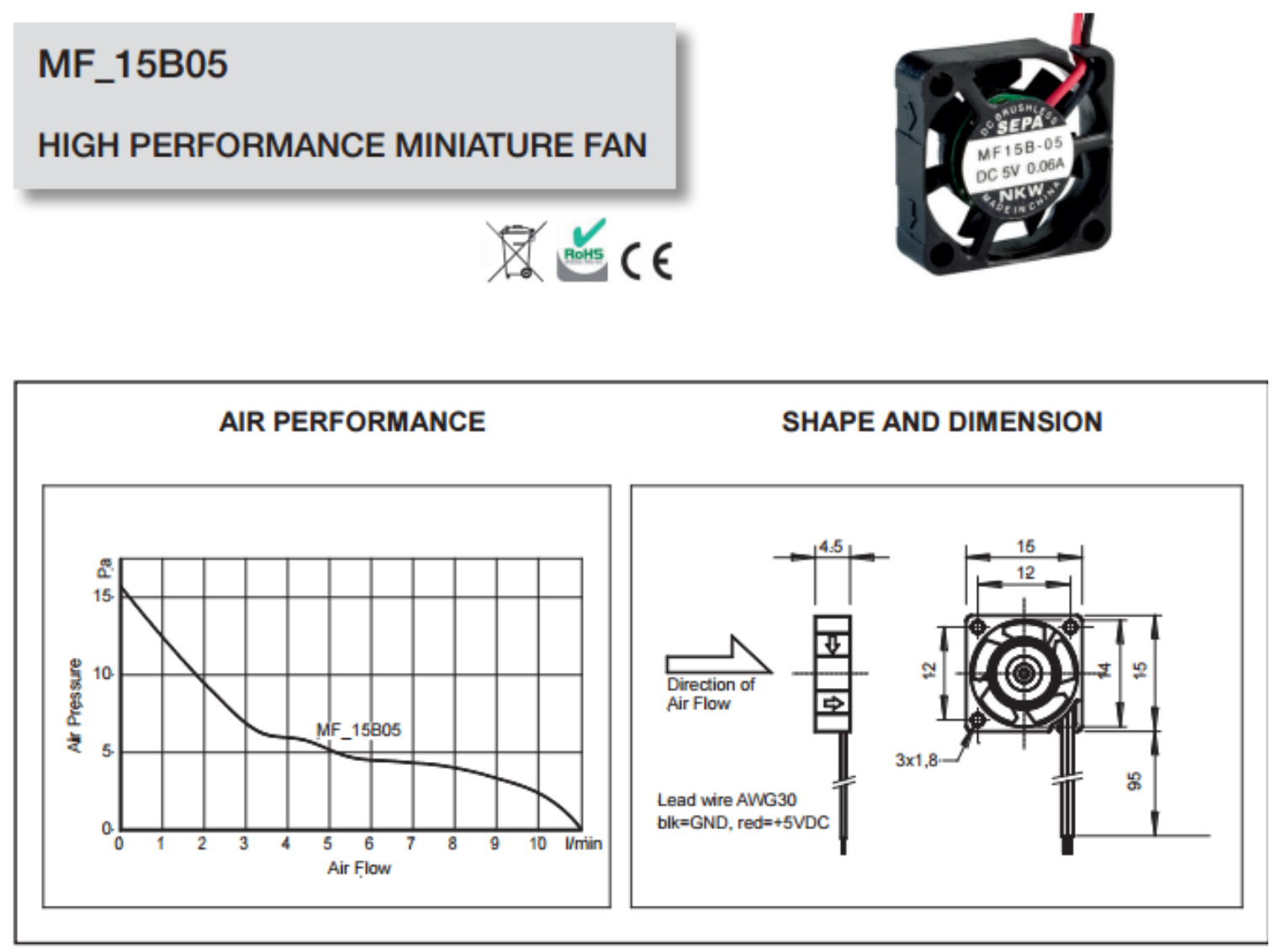

\section{PERFORMANCE}

\begin{tabular}{|c|c|c|}
\hline$C$ & & MF_15B05 \\
\hline Operating Voltage & {$[\mathrm{V}]$} & $4.5 \ldots 5 \ldots 5.5$ \\
\hline Typ. Operating Current & {$[\mathrm{mA}]$} & 60 \\
\hline Max. Start Current & {$[\mathrm{mA}]$} & 200 \\
\hline Max. Air Flow & {$\left[\mathrm{V} / \mathrm{min}-\mathrm{m}^{3} / \mathrm{h}\right]$} & $12-0.72$ \\
\hline Max. Air Pressure & {$\left[\mathrm{mmH}_{2} \mathrm{O}-\mathrm{Pa}\right]$} & $1.6-15.7$ \\
\hline Typ. Noise @1m & {$[\mathrm{dB}(\mathrm{A})]$} & 12 \\
\hline Typ. Rotor Speed & [RPM] & 13000 \\
\hline Operating Temperature & {$\left[{ }^{\circ} \mathrm{C}\right]$} & $-10 \ldots+60$ \\
\hline Life Expectancy $L_{10} / M T B F$ & [h] & $50000 / 140000 @ 20^{\circ} \mathrm{C}$ \\
\hline Bearing System & & sleeve bearing \\
\hline Weight & {$[\mathrm{g}]$} & 2 \\
\hline Packing Quantity & [PCs] & $50-100-500-1000$ \\
\hline
\end{tabular}

Figure 29 Fan Specification [35] 
HAMAMATESU

PHOTON IS OUR BUSINESS
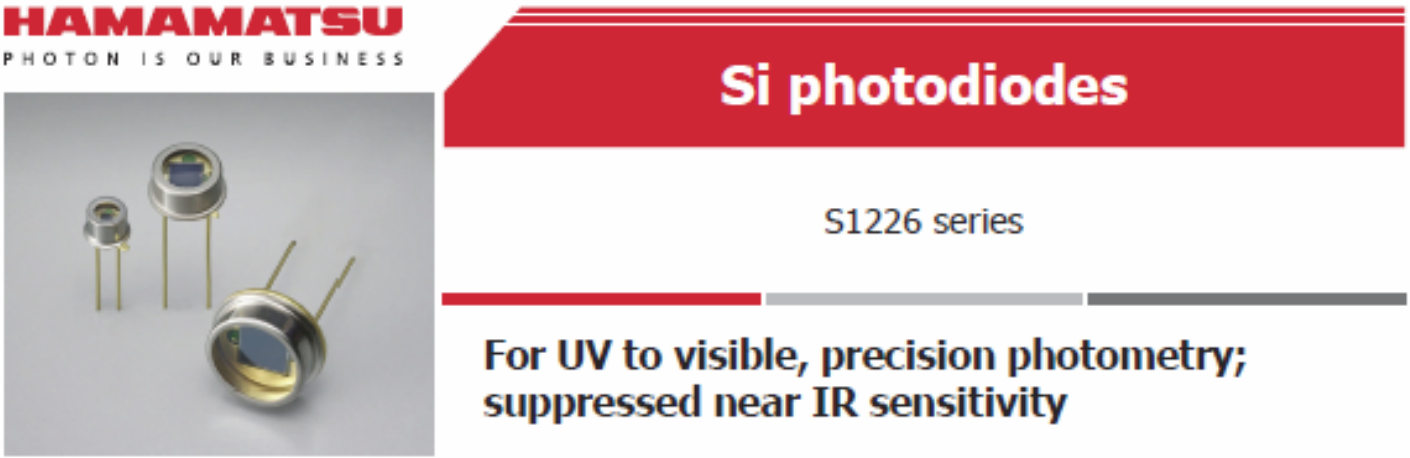

S1226 series

\section{For UV to visible, precision photometry; suppressed near IR sensitivity}

\section{- Features}

Suppressed near IR sensitivity

$\rightarrow$ High sensitivity in UV region (quartz glass type)

$\rightarrow$ Low dark current

$\Rightarrow$ High reliability

\section{- Applications}

Analytical equipment

$\Rightarrow$ Optical measurement equipment, etc.

- Structure / Absolute maximum ratings

\begin{tabular}{|c|c|c|c|c|c|c|}
\hline \multirow[b]{2}{*}{ Type no. } & \multirow[b]{2}{*}{$\begin{array}{l}\text { Dimensional } \\
\text { outline/ } \\
\text { Window } \\
\text { material" }\end{array}$} & \multirow[b]{2}{*}{ Package } & \multirow[b]{2}{*}{$\begin{array}{c}\text { Photosensitive } \\
\text { area size } \\
\text { (mm) }\end{array}$} & \multicolumn{3}{|c|}{ Absolute maximum ratings } \\
\hline & & & & $\begin{array}{l}\text { Reverse } \\
\text { voltage } \\
\text { VR max } \\
\text { (V) }\end{array}$ & $\begin{array}{c}\text { Operating } \\
\text { temperature } \\
\text { Topr } \\
\left({ }^{\circ} \mathrm{C}\right)\end{array}$ & $\begin{array}{c}\text { Storage } \\
\text { temperature } \\
\text { Tstg } \\
\left({ }^{\circ} \mathrm{C}\right)\end{array}$ \\
\hline S1226-18BQ & $(1) / Q$ & \multirow{2}{*}{ TO-18 } & \multirow{2}{*}{$1.1 \times 1.1$} & \multirow{8}{*}{1.7} & -20 to +60 & -55 to +80 \\
\hline S1226-18BK & $(2) / K$ & & & & -40 to +100 & -55 to +125 \\
\hline S1226-5BQ & $(3) / Q$ & \multirow{4}{*}{ TO-5 } & \multirow{2}{*}{$2.4 \times 2.4$} & & -20 to +60 & -55 to +80 \\
\hline S1226-5BK & $(4) / K$ & & & & -40 to +100 & -55 to +125 \\
\hline S1226-44BQ & $(5) / Q$ & & & & -20 to +60 & -55 to +80 \\
\hline S1226-44BK & $(6) / K$ & & & & -40 to +100 & -55 to +125 \\
\hline S1226-8BQ & $(7) / Q$ & \multirow{2}{*}{ TO-8 } & & & -20 to +60 & -55 to +80 \\
\hline S1226-8BK & $(8) / K$ & & $5.8 \times 5.8$ & & -40 to +100 & -55 to +125 \\
\hline
\end{tabular}

Note: Exceeding the absolute maximum ratings even momentarily may cause a drop in product quality. Always be sure to use the product within the absolute maximum ratings.

" Window material, $\mathrm{K}=$ borosilicate glass, $\mathrm{Q}=$ quartz glass

Electrical and optical characteristics (Typ. $\mathrm{Ta}=25^{\circ} \mathrm{C}$, unless otherwise noted)

\begin{tabular}{|c|c|c|c|c|c|c|c|c|c|c|c|c|c|c|c|}
\hline \multirow{3}{*}{ Type no. } & \multirow{3}{*}{$\begin{array}{c}\text { Spectral } \\
\text { response } \\
\text { range } \\
\lambda \\
\\
(\mathrm{nm}) \\
\end{array}$} & \multirow{3}{*}{ 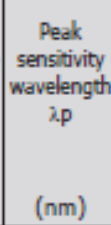 } & \multicolumn{4}{|c|}{$\begin{array}{c}\text { Photosensitivity } \\
\text { S } \\
\text { (A/W) }\end{array}$} & \multirow{2}{*}{\multicolumn{2}{|c|}{$\begin{array}{l}\text { Short } \\
\text { circuit } \\
\text { current } \\
\text { Isc } \\
100 \mathrm{ix}\end{array}$}} & \multirow{3}{*}{$\begin{array}{c}\text { Dark } \\
\text { current } \\
\text { Io } \\
\mathrm{VR}=10 \mathrm{mV} \\
\text { max. } \\
\\
\text { (pA) }\end{array}$} & \multirow{3}{*}{$\begin{array}{l}\text { Temp. } \\
\text { coefficient. } \\
\text { of } \mathrm{D} \\
\text { TCD } \\
\\
\\
\text { (times/ } /{ }^{\circ} \mathrm{C} \text { ) }\end{array}$} & \multirow{3}{*}{$\begin{array}{c}\text { Rise time } \\
\text { tr } \\
V_{R}=0 \mathrm{~V} \\
\mathrm{RL}=1 \mathrm{k} \Omega \\
\\
\text { (us) }\end{array}$} & \multirow{3}{*}{$\begin{array}{c}\text { Terminal } \\
\text { capactance } \\
\mathrm{c} \\
\mathrm{V}=0 \mathrm{~V} \\
\mathrm{f}=10 \mathrm{kHz} \\
\\
(\mathrm{pF})\end{array}$} & \multirow{2}{*}{\multicolumn{2}{|c|}{$\begin{array}{c}\text { Shunt } \\
\text { resistance } \\
\text { Rsh } \\
\text { VR=10 mV }\end{array}$}} & \multirow{3}{*}{$\begin{array}{c}\text { Noise } \\
\text { equivalent } \\
\text { power } \\
\text { NEP } \\
\\
\\
\left(\mathrm{W} / \mathrm{Hz}^{1 / 2}\right)\end{array}$} \\
\hline & & & \multirow{2}{*}{$\lambda p$} & \multicolumn{2}{|c|}{$200 \mathrm{~nm}$} & \multirow{2}{*}{\begin{tabular}{|c|} 
He-Ne \\
laser \\
633 \\
$\mathrm{~nm}$
\end{tabular}} & & & & & & & & & \\
\hline & & & & Min. & Typ. & & $\begin{array}{l}\text { Min, } \\
(\mu \mathrm{A})\end{array}$ & $\begin{array}{l}\text { Typ. } \\
(\mu \mathrm{A})\end{array}$ & & & & & $\begin{array}{l}\text { Min. } \\
(\mathrm{G} \Omega)\end{array}$ & $\begin{array}{l}\text { Typ. } \\
(\mathrm{G} \Omega)\end{array}$ & \\
\hline S1226-18BQ & 190 to 1000 & \multirow{8}{*}{720} & \multirow{8}{*}{0.36} & 0.10 & 0.12 & \multirow{8}{*}{0.34} & \multirow[t]{2}{*}{0.5} & \multirow[t]{2}{*}{0.66} & \multirow[t]{2}{*}{2} & \multirow{8}{*}{1.12} & \multirow[t]{4}{*}{0.15} & \multirow[t]{2}{*}{35} & 5 & 50 & \multirow{2}{*}{$1.6 \times 10^{-15}$} \\
\hline S1226-18BK & 320 to 1000 & & & \begin{tabular}{|l}
- \\
\end{tabular} & - & & & & & & & & 2 & & \\
\hline S1226-5BQ & 190 to 1000 & & & 0.10 & 0.12 & & \multirow{2}{*}{2.2} & \multirow{2}{*}{2.9} & \multirow{2}{*}{5} & & & 160 & 2 & 20 & $2.5 \times 10^{-15}$ \\
\hline S1226-5BK & 320 to 1000 & & & - & - & & & & & & & 100 & 2 & 20 & \\
\hline S1226-44BQ & 190 to 1000 & & & 0.10 & 0.12 & & \multirow{2}{*}{4.4} & \multirow{2}{*}{5.9} & \multirow{2}{*}{10} & & \multirow[t]{2}{*}{1} & \multirow{2}{*}{500} & \multirow[t]{2}{*}{1} & \multirow{2}{*}{10} & \multirow[t]{2}{*}{$3.6 \times 10^{-15}$} \\
\hline S1226-44BK & 320 to 1000 & & & - & - & & & & & & & & & & \\
\hline S1226-8BQ & 190 to 1000 & & & 0.10 & 0.12 & & \multirow{2}{*}{12} & 16 & 30 & & 2 & 1700 & 0.5 & 5 & $5.0 \times$ \\
\hline S1226-8BK & 320 to 1000 & & & - & - & & & 10 & 20 & & 2 & 1200 & 0.3 & 3 & \\
\hline
\end{tabular}

Figure 30 Photodiode Specification [15] 


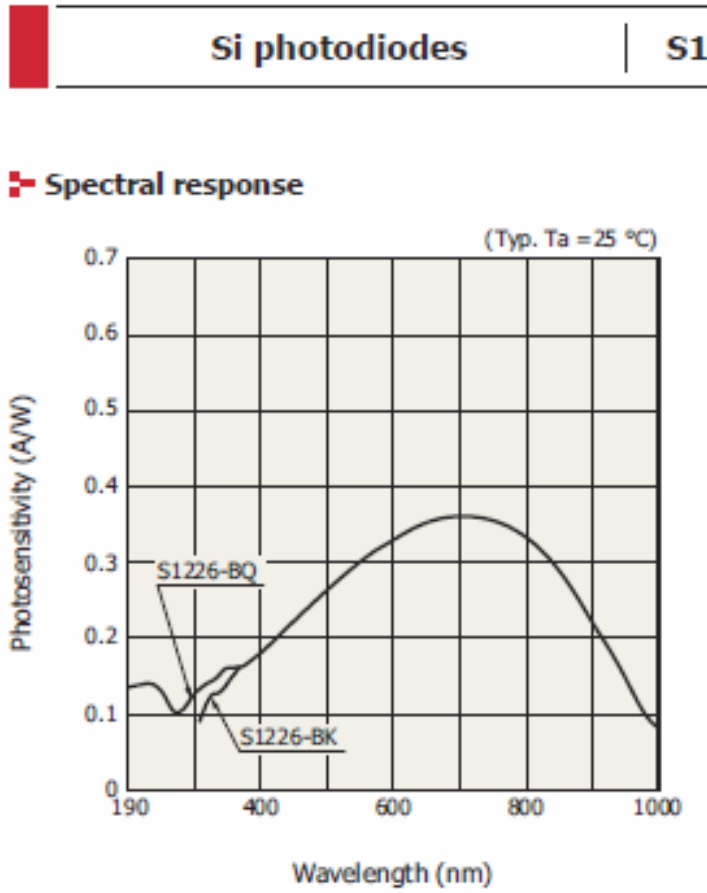

S1226 series

cocasias.

E- Photosensitivity temperature characteristic

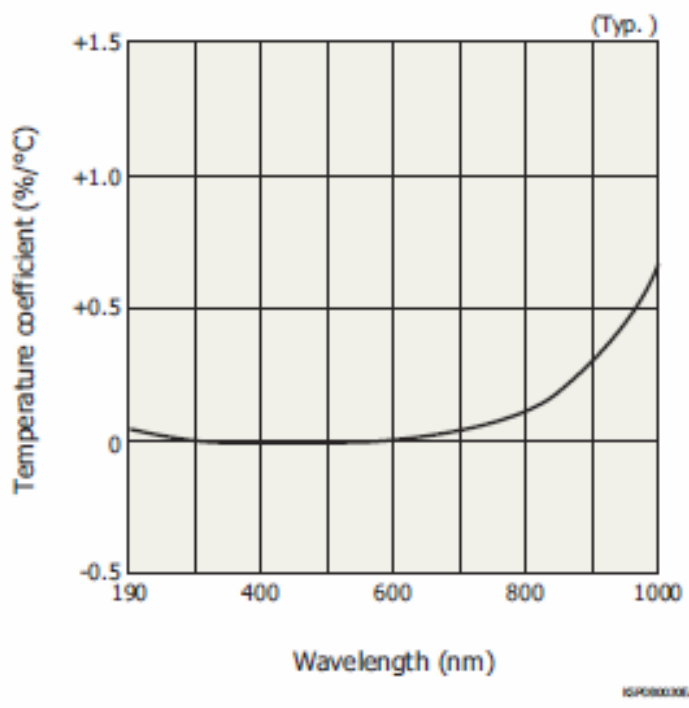

- Dimensional outlines (unit: $\mathbf{m m}$ )

(1) S1226-18BQ

- Dark current vs. reverse voltage

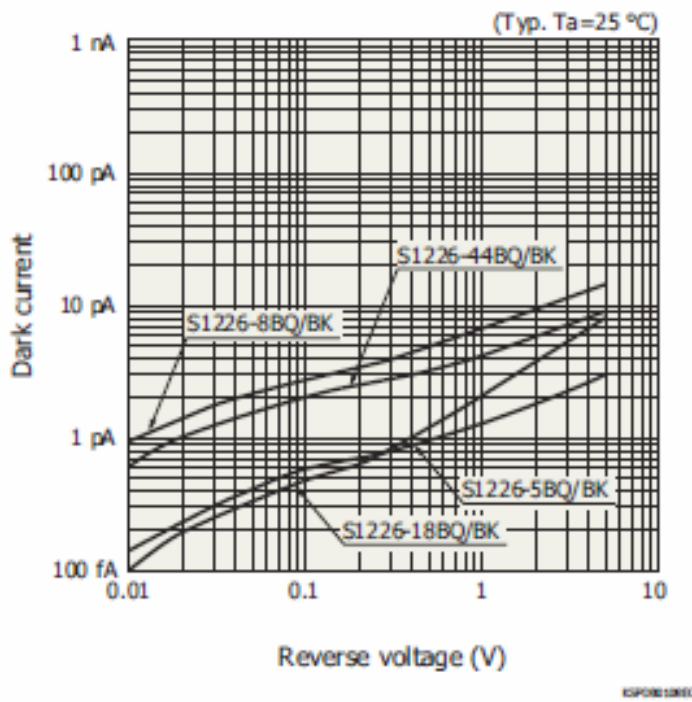

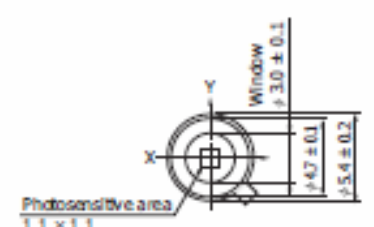

$1.1 \times 1.1$
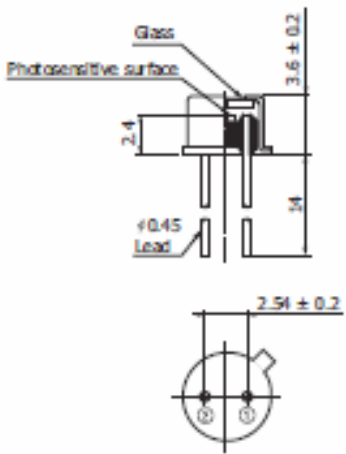

Connextad to caec

Distane from phowounative

arom conter to ap ontox

$0.3 x+2 s+0.3$

Figure 31 Photodiode Chart and Dimension Schematic [15] 


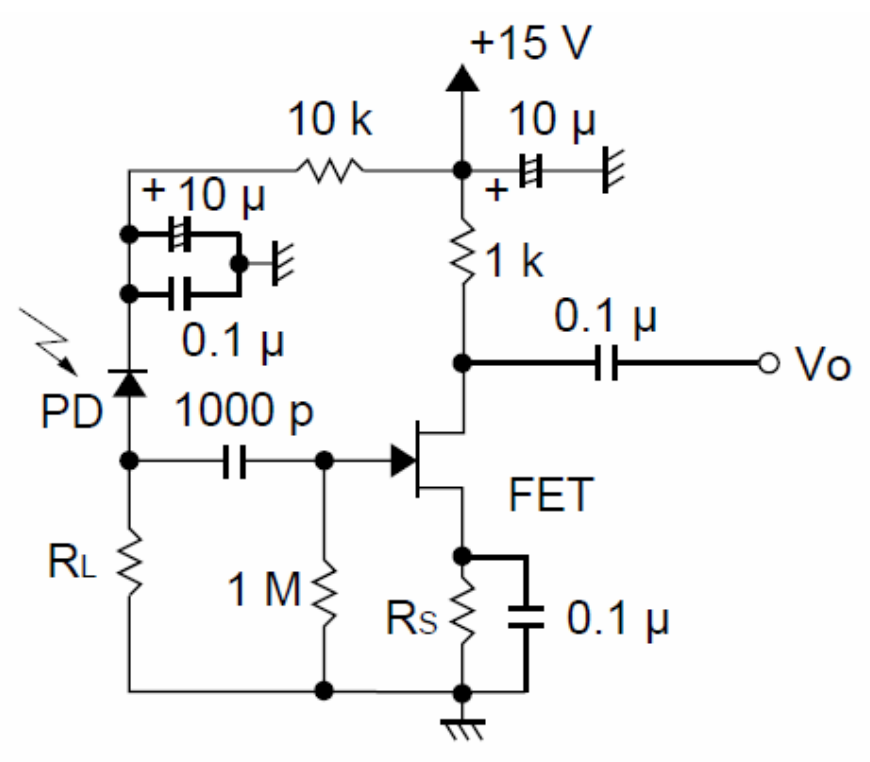

PD : High-speed PIN photodiodes (S5052, S2506-02, S5971, S5972, S5973, etc.)

$\mathrm{RL} \quad$ : Determined by sensitivity and time constant of $\mathrm{Ct}$ of photodiode

Rs : Determined by operation point of FET

FET: 2SK152, 2SK192A, 2SK362, etc.

Figure 32 Schematic of a Low Noise Light-Sensitive Preamplifier Circuit by

Hamamatsu [36] 
